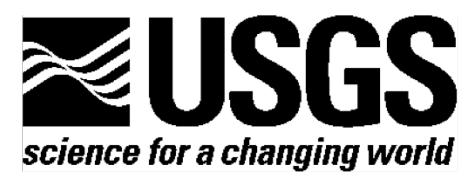

\title{
ShakeNet: A Portable Wireless Sensor Network for Instrumenting Large Civil Structures
}

By Monica D. Kohler, Shuai Hao, Nilesh Mishra, Ramesh Govindan, and Robert Nigbor

Open-File Report 2015-1134

U.S. Department of the Interior

U.S. Geological Survey 


\section{U.S. Department of the Interior \\ SALLY JEWELL, Secretary}

\section{U.S. Geological Survey \\ Suzette M. Kimball, Acting Director}

U.S. Geological Survey, Reston, Virginia: 2015

For more information on the USGS-the Federal source for science about the Earth,

its natural and living resources, natural hazards, and the environment-visit

http://www.usgs.gov/ or call 1-888-ASK-USGS (1-888-275-8747).

For an overview of USGS information products, including maps, imagery, and publications, visit http://www.usgs.gov/pubprod/.

Any use of trade, firm, or product names is for descriptive purposes only and does not imply endorsement by the U.S. Government.

Although this information product, for the most part, is in the public domain, it also may contain copyrighted materials as noted in the text. Permission to reproduce copyrighted items must be secured from the copyright owner.

Suggested citation:

Kohler, M.D., Hao, Shuai, Mishra, Nilesh, Govindan, Ramesh, and Nigbor, Robert, 2015, ShakeNet-A portable wireless sensor network for instrumenting large civil structures: U.S. Geological Survey Open-File Report 20151134, 31 p., http://dx.doi.org/10.3133/ofr20151134.

ISSN 2331-1258 (online) 


\section{Acknowledgments}

This work was funded by U.S. Geological Survey National Earthquakes Hazards Reduction Program Award Numbers G09AP00101 and G09AP00102. We acknowledge intellectual support from the Reftek engineers who were involved at every level of these development efforts. We are also grateful for reviews from John Evans and Tat Fu and editing by Jessica Dyke that greatly strengthened this report. 


\section{Contents}

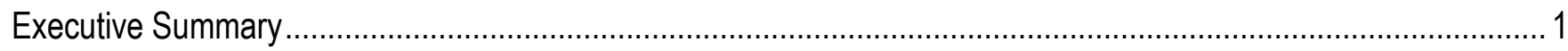

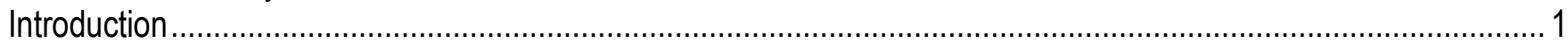

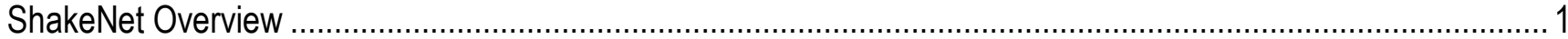

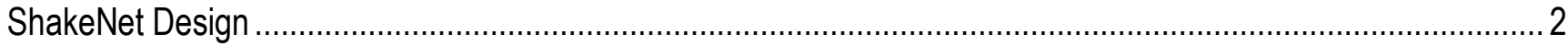

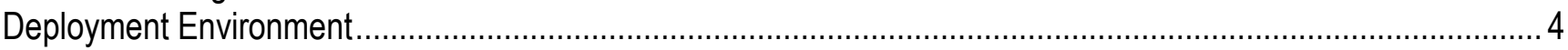

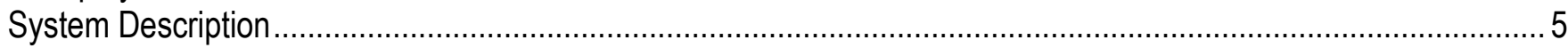

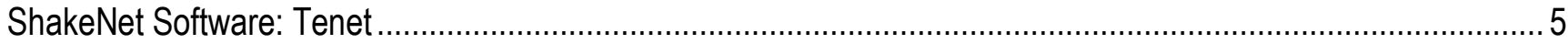

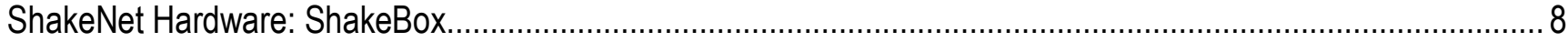

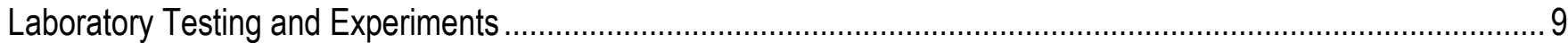

FFT (Fast Fourier Transform) and Channel Analysis With Function Generator ................................................ 9

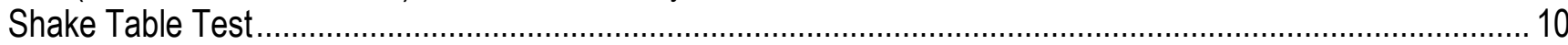

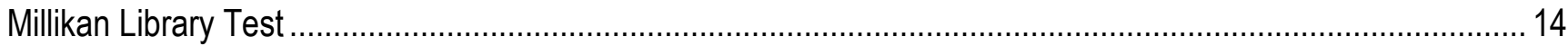

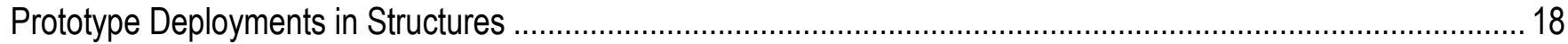

Deployment of Instruments During Early Development.............................................................................. 18

ShakeNet Prototype Deployments ………………………..................................................................... 19

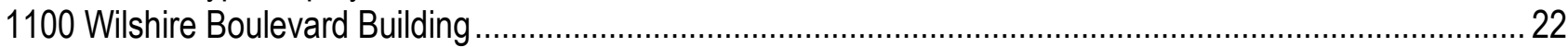

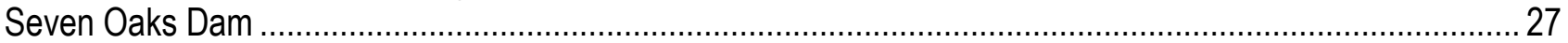

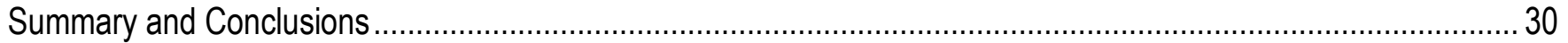

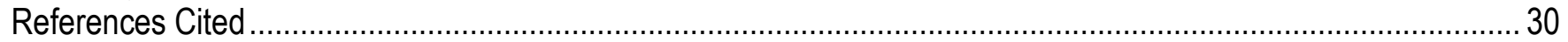

\section{Figures}

1. ShakeBox hardware block diagram. ADC, Analog-to-Digital Converter. I2C, Inter-Integrate Circuit. The imote-2 is also referred to as the Imote2 in the text.

2. Schematic illustrating Tenet architecture. A lower tier consists of motes and an upper tier consists of masters. MAC, medium access control. Tenet incorporates the Flooding Time Synchronization Protocol (FTSP) for time synchronization.

3. Photographs of the ShakeBox components. $A$, ShakeBox with weatherproof enclosure; six-inch ruler shown for scale. B, Placement of modules with 6 -inch ruler shown for scale. $C$, Connection between individual modules: a, Central Processing Unit (CPU) module with Imote2; b, power module; c, Analog-toDigital Converter (ADC) module.

4. Graphs showing FFT (Fast Fourier Transform) analysis of input function generator frequency sweep from 0.2 to $125 \mathrm{~Hz}$ (hertz) with $1 \mathrm{Vpp}$ (volt peak-to-peak) amplitude. Negative frequencies are also plotted by default......

5. Photographs showing shake table equipment and instrumentation setup. $A$, Shake table top showing mounted steel plate with Dytran piezoelectric and Colibrys MEMS accelerometers, and additional ShakeBox components on chair. B, Closeup of mounted plate with accelerometers. C, 24-bit digitizer and $P C$ recording setup for Dytran accelerometers.

6. A, Shake table test results comparing ShakeBox (Colibrys MEMS: black curves) with Dytran piezoelectric accelerometer response (red curves), recorded at 100 sps. Shaking was performed in the Colibrys Channel 2 direction with $1 \mathrm{~Hz}$ sine wave input..

6. $B$, Shake table test results comparing ShakeBox (Colibrys MEMS: black curves) with Dytran piezoelectric accelerometer response (red curves), recorded at 100 sps. Shaking was performed in the Colibrys Channel 2 direction with $5 \mathrm{~Hz}$ sine wave input.. 
6. C, Shake table test results comparing ShakeBox (Colibrys MEMS: black curves) with Dytran piezoelectric accelerometer response (red curves), recorded at 100 sps. Shaking was performed in the Colibrys Channel 2 direction with $25 \mathrm{~Hz}$ sine wave input

7. Photographs showing Robert A. Millikan Memorial Library, shaker, and sensors that were tested. A, Robert A. Millikan Memorial Library on the Caltech campus, viewed from the northeast. $B$, Kinemetrics VG-1 eccentric-mass shaker located on the roof of Millikan Library. The counter-rotating buckets, shown empty, can be loaded with different configurations of lead weights to impart different forces on the building. $C$, Accelerometers located in the basement that were recording during the Millikan shake test. The ShakeBox is the small black box with the orange triangle symbol on top, behind the blue box.

8. A, Graphs showing acceleration responses due to forced vibrations recorded by the ShakeNet ShakeBoxes collocated with an Episensor in the basement of Millikan Library, California Institute of Technology.

8. $B$, Graphs showing acceleration responses due to forced vibrations recorded by the ShakeBoxes collocated with an Episensor on the 9th floor of Millikan Library, California Institute of Technology... 18

9. A, Vincent Thomas Bridge, Los Angeles Harbor, California. B, ShakeNet prototype deployment across part of the bridge deck.

10. Graph showing accelerations recorded during the deployment at Vincent Thomas Bridge, Los Angeles, California, in July 2006. Vertical accelerations have been bandpass filtered for frequencies between 0.2 and $5.0 \mathrm{~Hz}$

10. Graph showing spectra of accelerations recorded during the deployment at the Vincent Thomas Bridge, Los Angeles, California, in July 2006.

11. Photo and SketchUp ${ }^{\mathrm{TM}}$ diagram of the 1100 Wilshire Boulevard building in Los Angeles, California.......... 23

12. A, Spectra from a 1 -hour section of horizontal component waveforms from 30 ShakeBoxes deployed in stairwells on various floors arranged from bottom to top of building (bottom to top of figure) at 1100 Wilshire Boulevard, Los Angeles, California. Numbers overwriting file name in left-hand column indicate amplitude normalization factor.

12. B, Spectrogram from a section of horizontal component data recorded by a ShakeBox deployed on the $36^{\text {th }}$ floor, from the test deployment in the building at 1100 Wilshire Boulevard, Los Angeles, California.

13. Seven Oaks Dam in Highland, California. A, Aerial view. Picture taken on February 8, 2005, by Steve Schumaker. B, Example ShakeBoxes deployed on crest of dam. $C$, Network configuration showing locations (red squares) of the 31 seismometers

14. Ambient vibration spectra from transverse direction (channel 2, north-south orientation) produced from several hours of acceleration data recorded during one of the deployment days at Seven Oaks Dam in Highland, California. Numbers overwriting file name in left-hand column indicate amplitude normalization factor.

\section{Tables}

1. Floors with sensors in the 1100 Wilshire Boulevard building deployment, Los Angeles, California 24

2. Locations of sensors and associated clusters during Seven Oaks Dam deployment. Sensors on crest road are listed in increasing distance order from westernmost to easternmost end and are approximately 120 feet apart. See also figure 13. 


\begin{tabular}{ll}
\multicolumn{2}{l}{ Abbreviations } \\
ADC & Analog-to-Digital Converter \\
ANSS & Advanced National Seismic System \\
BOINC & Berkeley Open Infrastructure for Network Computing \\
CPU & Central Processing Unit \\
CSN & Community Seismic Network \\
EEPROM & Electrically Erasable Programmable Read-Only Memory \\
FPGA & Field-Programmable Gate Array \\
FTSP & Flooding Time Synchronization Protocol \\
GPS & Global Positioning System \\
JTAG & Joint Test Action Group \\
LED & Light Emitting Diode \\
MAC & Medium Access Control \\
MDA & Mono Class D Amplifier \\
MEMS & Micro-electroMechanical Systems \\
NTP & Network Time Protocol \\
QCN & Quake-Catcher Network \\
Reftek & Refraction Technology, Inc. \\
SDRAM & Synchronous Dynamic Random Access Memory \\
SEL & Module Selection \\
SPI & Serial Peripheral Interface \\
USB & Universal Serial Board \\
USGS & U.S. Geological Survey \\
&
\end{tabular}




\title{
ShakeNet: A Portable Wireless Sensor Network for Instrumenting Large Civil Structures
}

\author{
By Monica D. Kohler, ${ }^{1}$ Shuai Hao, ${ }^{2}$ Nilesh Mishra, ${ }^{2}$ Ramesh Govindan, ${ }^{2}$ and Robert Nigbor ${ }^{3}$
}

\section{Executive Summary}

We report our findings from a U.S. Geological Survey (USGS) National Earthquake Hazards Reduction Program-funded project to develop and test a wireless, portable, strong-motion network of up to 40 triaxial accelerometers for structural health monitoring. The overall goal of the project was to record ambient vibrations for several days from USGS-instrumented structures. Structural health monitoring has important applications in fields like civil engineering and the study of earthquakes. The emergence of wireless sensor networks provides a promising means to such applications. However, while most wireless sensor networks are still in the experimentation stage, very few take into consideration the realistic earthquake engineering application requirements. To collect comprehensive data for structural health monitoring for civil engineers, high-resolution vibration sensors and sufficient sampling rates should be adopted, which makes it challenging for current wireless sensor network technology in the following ways: processing capabilities, storage limit, and communication bandwidth. The wireless sensor network has to meet expectations set by wired sensor devices prevalent in the structural health monitoring community. For this project, we built and tested an application-realistic, commercially based, portable, wireless sensor network called ShakeNet for instrumentation of large civil structures, especially for buildings, bridges, or dams after earthquakes. Two to three people can deploy ShakeNet sensors within hours after an earthquake to measure the structural response of the building or bridge during aftershocks. ShakeNet involved the development of a new sensing platform (ShakeBox) running a software suite for networking, data collection, and monitoring. Deployments reported here on a tall building and a large dam were real-world tests of ShakeNet operation, and helped to refine both hardware and software.

\section{Introduction}

\section{ShakeNet Overview}

ShakeNet is a multi-tier, portable, vibration sensing system of tens of wireless nodes that can be rapidly deployed by two to three people on large civil structures, for example, tens of floors of a large building or tens of locations on a long bridge. It was designed to collect structural vibration measurements for up to a week from each node within the network. This portable system can be used to quickly (within 3-5 hours) instrument large structures immediately after an earthquake to capture

\footnotetext{
${ }^{1}$ California Institute of Technology

${ }^{2}$ University of Southern California

${ }^{3}$ University of California, Los Angeles
} 
aftershock and ambient motions. Because ShakeNet is portable, it can also be used at other times to understand the structural properties of different classes of structures (steel-frame versus reinforcedconcrete buildings). Each of the wireless sensor nodes (called ShakeBoxes) is equipped with a 24-bit analog-to-digital conversion board supporting high dynamic range triaxial accelerometers suitable for Advanced National Seismic System (ANSS) Class A (Advanced National Seismic System Structural Instrumentation Guideline Committee, 2005; Advanced National Seismic System Technical Integration Committee, 2007) seismic strong-motion sensing. The system comes preloaded with sensing software as well as deployment tools that enable structural engineers to rapidly deploy the network. In addition to the sensors, the system contains 10 to 15 master-tier nodes (embedded single-board computers) that provide more robust communications capacity. This wireless strong-motion network was specifically designed to deploy in structures with no existing power or communications infrastructure. It conforms to most ANSS Class A strong-motion design specifications, the most accurate and advanced class of structural measurement systems.

To test ShakeNet, we performed large-scale deployments on two USGS-instrumented structures: (1) the 1100 Wilshire Boulevard building in downtown Los Angeles, California, and (2) Seven Oaks Dam in Redlands, California. These large-scale deployments assisted with the development and tuning of the hardware and software in ShakeNet, and also demonstrated the strengths and weakness of such a wireless quick-deployment system.

Experience with these two dense structural arrays illustrates the value of collecting a large database of pre-event data on structures to use in future analyses of large-magnitude earthquake shaking data. While the responses of large structures in normal conditions are close to noise levels, even ambient vibrations and small earthquakes can provoke sufficient structural responses to detect traveling wave and modal structural properties useful for system identification purposes, for example, in the steel moment-frame UCLA (University of California, Los Angeles) Factor building (Kohler and others, 2005, 2006, 2007). The Millikan Library on the California Institute of Technology (Caltech) campus exhibits changes in modal parameters through time as a result of large ground shaking; Clinton and others (2006) report deviations in natural frequencies from the study of continuous monitoring of the $9^{\text {th }}$ floor of Millikan Library. These studies illustrate the importance of understanding and documenting the dynamic properties of different classes of structures (steel-frame versus reinforced-concrete buildings) within the linear response regime before nonlinear response might occur.

\section{ShakeNet Design}

ShakeNet was motivated by earlier work on instrumenting a long-span suspension bridge, the Vincent Thomas Bridge at the entrance to the Los Angeles Harbor, with wireless sensors. For the experiment, twenty wireless sensors were deployed on the bridge, and the sensor network acquired vibration samples continuously from each sensor for 24 hours. Although the results from the experiment were encouraging in terms of quick deployment in a matter of hours, and the structural characteristics derived from the collected data were consistent with previously published results, a few shortcomings were evident. The mono (single-channel) amplifier MDA-400 vibration card with 16-bit analog-todigital converter (ADC) used for recording the vibrations was not suitable for capturing low (sub-1 hertz [Hz]) fundamental frequencies of large structures. As structure (buildings, bridges) sizes increase, we wish to record lower fundamental frequencies associated with their natural frequencies of vibration because such low-frequency responses are of interest for structural analysis. In addition, the offsite development and preparation time required for the Vincent Thomas Bridge deployment was substantial.

Although we were able to extract macroscopic structural properties such as the modal frequencies, the specific board we used (the MDA-400 from Crossbow) had several shortcomings. It 
had only 16-bit resolution; this resolution is inadequate for monitoring ambient vibrations in large structures. It was originally designed for high-frequency sensing in the $\mathrm{kHz}$ (kilohertz) range, so its response at the sub-1 Hz modal frequencies of large structures was poor. It had a hardware fault which resulted in a signal offset that caused signal clipping at high amplitudes. Finally, the board was designed to interface only with a limited set of accelerometers, none of which was well-suited to structural sensing. A better accelerometer with higher signal-to-noise ratio and sensitivity was needed for structural health monitoring.

ShakeNet development addressed the shortcomings of the earlier system which was based on more industrial components. Although much of ShakeNet hardware was constructed using commercial off-the-shelf components (sensor network nodes known as motes and embedded single-board computers known as masters), a renewed focus was placed on the specific requirements for earthquake and ambient vibrations in large civil structures. What made ShakeNet application-realistic was our careful attention to the design of its data-acquisition hardware, the ShakeBoard (fig. 1). The ShakeBoard is a highresolution (24-bit) acceleration data acquisition system that uses commercial low-noise MicroelectroMechanical Systems (MEMS) accelerometers and conforms to Class A resolution specifications established by the U.S. Geological Survey for their Advanced National Seismic System (Advanced National Seismic System, 2005, 2007). To our knowledge, no comparable device exists on the market today.

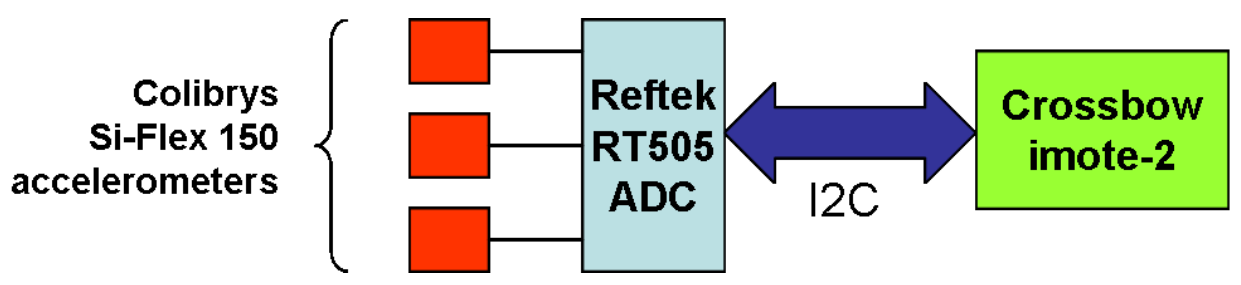

Figure 1. ShakeBox hardware block diagram. ADC, Analog-to-Digital Converter. I2C, Inter-Integrate Circuit. The imote- 2 is also referred to as the Imote 2 in the text.

ShakeNet can be rapidly deployed on several floors of a large building or multiple locations on a large bridge or dam with no dependence on existing power or communications infrastructure. It was designed to collect structural vibration measurements for up to a week from each node within the network. This portable system can be used to instrument large structures within hours after an earthquake. Since there will be a need to deploy multiple dense structural networks rapidly after a large earthquake has occurred, this network uses innovative hardware and software design to accommodate fast deployment by only two or three people. The network consists of two levels of complexity that make network reconfiguration based on suspected damage locations easier (Gnawali and others, 2006). The higher-level nodes include a processor on which the algorithms run continuously, reporting back to a central processing unit, for example the engineer's office computer (master nodes). Lower, more primitive nodes consist of the sensor and digitizer (motes), but these are in constant communication with at least one higher-level node. The results of the networking software are used to redeploy nodes during aftershock sequences in areas where significant damage is suspected.

The ShakeBoard hardware consists of a low-power analog-to-digital converter (ADC) board developed by RefTek (http://www.reftek.com). This board provides a 24-bit delta-sigma modulator at 250 sps (samples per second). Internally, the board uses a Cirrus Logic analog modulator, together with a digital filter. The sample rate and filter coefficients are all programmable. This board interfaces with three Si-Flex 150 accelerometers from Colibrys Inc. and talks to a Crossbow Imote2 over the Serial 
Peripheral Interface (SPI) bus. The accelerometers are among the most accurate, low-noise MEMS devices on the market. They provide full-scale $\pm 3 \mathrm{~g}$ (units of gravitational acceleration) measurements with a dynamic range exceeding $120 \mathrm{~dB}$ (decibels). The Imote2 has a 32-bit Intel processor, $32 \mathrm{MB}$ (megabytes) of SDRAM (Synchronous Dynamic Random Access Memory), and $32 \mathrm{MB}$ of flash. It uses an 802.15.4-compliant radio with a nominal data rate of $250 \mathrm{Kbps}$ (kilobits per second). The total power consumption of the unit is about $750 \mathrm{~mW}$ (milliwatts). All ShakeNet hardware conforms to ANSS Class A strong-motion design specifications for amplitude and timing resolution (Advanced National Seismic System Structural Instrumentation Guideline Committee, 2005; Advanced National Seismic System Technical Integration Committee, 2007).

The master level nodes (masters) consist of an embedded single board computer running on the Linux operating system and store data on an $8 \mathrm{~GB}$ (gigabytes) compact flash drive. The masters communicate to the ShakeBoxes via a Crossbow Tmote sensor network mote connected to one of the master's USB (Universal Serial Board) ports. The Tmote acts as a modem which transmits packets coming from the master node over the 802.15.4 radio. Similarly, it sends packets received from the ShakeBoxes to the master over USB. For our deployments, we used two different makes of masters: Eboxes and Habey.

ShakeNet's software was built upon Tenet (Gnawali and others, 2006). Tenet is a programmable wireless sensing software algorithm designed for multi-tier sensor networks. In Tenet, applications run on a tier of more capable nodes (the masters) and task low-power sensor nodes to collect and conduct elementary processing of data. Tenet simplifies the development of sensing applications and can run multiple applications concurrently. Prior to our work, the Tenet research prototype was not robust enough for week-long deployments and did not include tools for rapid deployment. The experience from our deployments helped us develop a production-quality version of Tenet for ShakeNet. We also added tools to support rapid ShakeNet deployment. To this end, we developed (1) lightweight network monitoring tools that troubleshoot network topology and routing, or deduce the network capacity of a particular deployment; (2) mobile, handheld management consoles that simplified the task of iteratively deploying nodes; and (3) remote-monitoring tools that were used to detect sensor or network failures postdeployment.

\section{Deployment Environment}

ShakeNet deployment requires placing the nodes in difficult radio environments, either inside large structures with non-radio-transparent structural material or on large outdoor structures with large transmission distances, often on radio-reflective structural materials. ShakeNet requires the communication protocol to take care of packet drops and find a route to the data collection node. Development of robust, working protocols for these operations from scratch required considerable time and expertise. We do not envision the end users of ShakeNet to write wireless sensor network data collection and communication protocols. Use of existing wireless sensor network software and modifying it for ShakeNet's requirements reduces the development time. Use of multiple masters to handle relatively small numbers of nodes also helps with slow networks that may saturate easily with overhead associated with larger numbers of nodes. We also needed a wireless sensor network that could be tasked to operate a number of applications. It needed to have tools that could help in rapid application development as well as for rapid deployment in the field. The Tenet software, discussed below, fulfilled a number of these requirements and, thus, was used to develop the software suite required to run ShakeNet over the ShakeBoxes. 


\section{System Description}

\section{ShakeNet Software: Tenet}

The software for running ShakeNet was built using the Tenet architecture for tiered embedded networks. Tenet is based on the observation that for scalability, modern sensor network deployments ideally have two tiers: a lower tier consisting of motes that enable flexible deployment of dense instrumentation and an upper tier containing fewer, relatively less-constrained 32-bit nodes with higherbandwidth radios, called masters (fig. 2). Tenet constrains the placement of application functionality in a sensor network according to the principle that multi-node data fusion functionality and multi-node application logic should be implemented only in the master tier. The cost and complexity of implementing this functionality in a fully distributed fashion in the motes outweighs the performance benefits of doing so. This tiered design is fundamental to scaling the size and spatial extent of sensor networks because the masters collectively have greater network capacity and a larger spatial reach than a flat (non-tiered) field of motes. We ported the Tenet software suite for running over the Imote2 (the mote used in the ShakeBoxes; sometimes referred to as an imote-2) and added additional features to run ShakeNet.

\section{The Tenet Architecture}

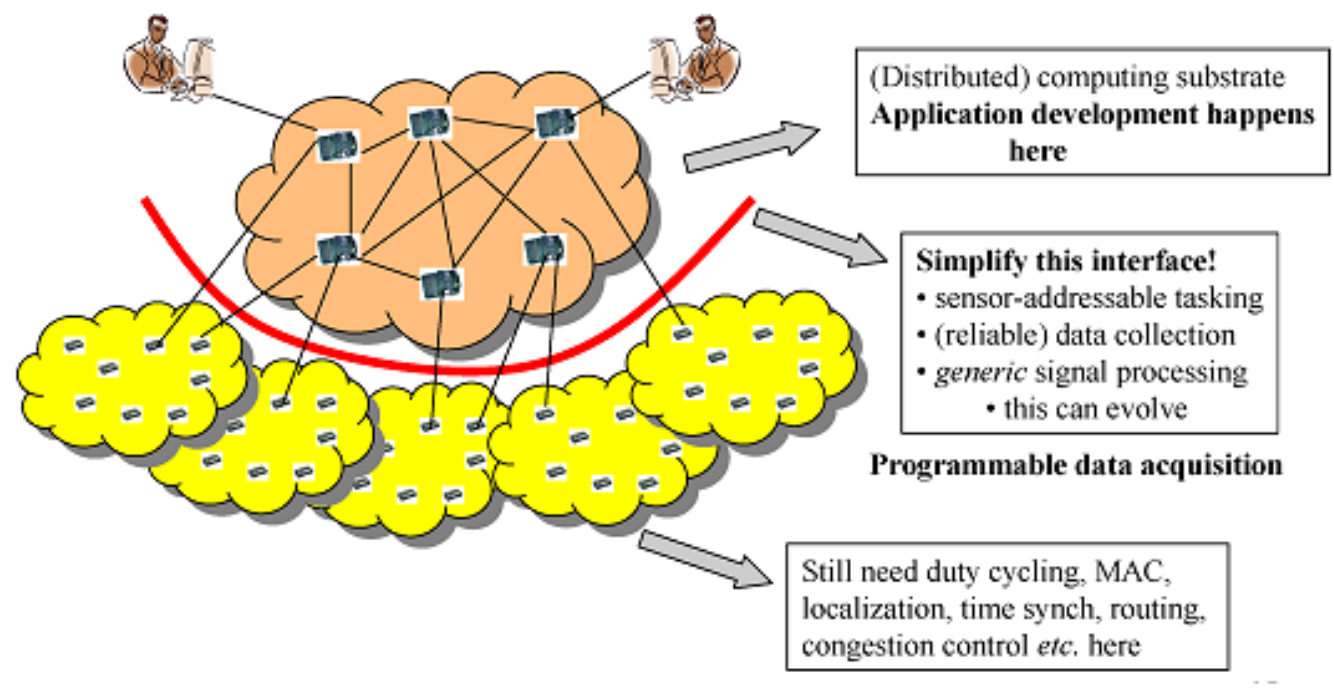

Figure 2. Schematic illustrating Tenet architecture. A lower tier consists of motes and an upper tier consists of masters. MAC, medium access control. Tenet incorporates the Flooding Time Synchronization Protocol (FTSP) for time synchronization.

Because the computation and storage capabilities of masters are likely to be at least an order of magnitude higher than the motes at any point in the technology curve, masters are the more natural candidates for data fusion and higher-level analysis. The principle allows motes to process locally generated sensor data (that is, data collected at the mote) such as decimation by the ADC; this can result in significant communication energy savings. This architectural principle simplifies application 
development, resulting in a generic mote-tier networking subsystem that only needs to be implemented once. It can be reused for a variety of applications, simplifying mote functionality, thereby enabling energy-efficient mote operation, all without significant loss, and with significant gains in overall system efficiency.

In Tenet, applications run on one or more master nodes. They task motes to sense and locally process data. Conceptually, a task is a small program written in a domain-specific language. The results of mote tasks are delivered by the Tenet system to the application program. This program can then fuse the returned results and either retask the motes or trigger other sensing modalities. More than one application can run concurrently on Tenet. Our Tenet system adheres to the Tenet architectural principle by constraining mote functionality. In our system, all communication from an application to the mote tier must be in the form of tasks, and applications have no way of expressing multi-node fusion computation to be performed in the mote tier.

ShakeNet data collection happens using the Tenet hierarchical architecture. We have a higher tier of master nodes, which task the nodes and collect the data responses coming from them. The current prototype is able to collect data from two ShakeBoxes sampling data at 125 sps under each master, at which point we saturate the available radio bandwidth and cannot add any more ShakeBoxes under this master. To overcome this limitation, we implemented the Steim2 algorithm for compressing data on each ShakeBox prior to sending it to the master.

Tenet is quite stable for a research prototype, largely because we have invested several personmonths testing the code in preparation for a bridge deployment. However, that deployment was restricted in two important ways. First, we were not confident of the dynamic routing protocols both on the master and mote tiers. We did not use dynamic routing software because it was not reliable at the time of deployment. In laboratory experiments prior to deployment, we noticed routing pathologies and decided to instead use static routing in our deployment. Although we were lucky not to have had node failures during our one-day experiment, we cannot rely on the same design for a week-long deployment. Second, our deployment did not use Tenet's time synchronization. Tenet incorporates the Flooding Time Synchronization Protocol (FTSP) (Maroti and others, 2004) implementation because we observed problems similar to those described in Werner-Allen and others (2006) during laboratory tests. FTSP is a time synchronization protocol optimized for wireless environments and outperforms Network Time Protocol (NTP) in these environments. FTSP results in high-precision time on resource-limited wireless networks by using periodic flooding of time synchronization messages.

The overall goals for ShakeNet included the following requirements of the ShakeBox:

- Resolution: We required that the ShakeBox conform to the specifications of a Class A strongmotion accelerometer system (Advanced National Seismic System Structural Instrumentation Guideline Committee, 2005; Advanced National Seismic System Technical Integration Committee, 2007). A Class A device is defined to have a resolution of greater than $111 \mathrm{~dB}$ or, noise equivalently, less than $6.7 \mu g$ (micro- $g$ where $g$ is units of gravitational acceleration). These specifications translate to a 24-bit device that provides at least 20 bits of usable resolution over $\pm 4 \mathrm{~g}$ of acceleration. The Class A device is the most accurate strong-motion accelerometer defined by the ANSS standards.

- Form Factor: We required that the form factor of the sensor node (together with the ShakeBox) be such that 75 of them will fit in a small number (say 5-7) of commercially available mobile work boxes. This requirement enables a small number of people (two to three) to quickly transport these devices and install them in a building. We placed no form factor requirement on the master tier nodes. We assume that these nodes can be placed conveniently in locations where there is building power (because these nodes will not have attached sensors, there is greater 
leeway in their placement); without batteries, most embedded 32-bit devices have a form factor comparable to a netbook. Alternatively, we conducted a multiple-day deployment in an external environment with master tier node power supplied by car batteries.

- Energy Efficiency: We required that each node be able to sustain a week-long deployment with continuous sensing at up to 100 sps of three-axis measurements. As we have discussed previously, this requirement is motivated by the expected duration of major aftershocks. Clearly, there is a trade-off between form factor and duration of recording: A longer lifetime requirement may necessitate larger devices. We discuss below a preliminary design that represents an acceptable compromise between lifetime and form factor.

- ShakeBox Hardware: The ShakeBox hardware was developed by a commercial entity, Refraction Technology Inc. (Reftek). At the core of the ShakeBox is an analog-to-digital converter (ADC) board called the RT505, a proprietary low-power ADC developed by Reftek. This board provides a 24-bit delta-sigma modulator at 250 sps. Internally, the board uses a Cirrus Logic CS5372 analog modulator, together with a CS5322 digital filter. The sampling rate and filter coefficients are all programmable. This board interfaces with three Si-Flex 150 accelerometers from Colibrys Inc. and talks to a Crossbow Imote2 over the SPI bus. The accelerometers are among the most accurate and low-noise MEMS devices on the market. They provide full-scale $\pm 3 \mathrm{~g}$ measurements with a dynamic range of $120 \mathrm{~dB}$. The Imote 2 is a highperformance sensor device whose design was originally motivated by high-frequency sensing applications (Nagayama and others, 2007). It has an Intel PXA271 processor, $32 \mathrm{MB}$ of SDRAM, and 32 MB of flash memory. Finally, it uses the CC2420 802.15.4-compliant radio with a nominal data rate of $250 \mathrm{Kbps}$.

- The Driver: The communication protocol defines how each module should behave for information exchange. The driver implements the protocol according to the specifications. With the driver, the CPU (Central Processing Unit) module can turn the power and sensor module on and off, and configure them. The driver supports the following functionalities: (1) select a specified device on any of the modules, (2) read from and write to FPGA (Field-Programmable Gate Array) registers and the EEPROM (Electrically Erasable Programmable Read-Only Memory) on any of the modules, and (3) collect sample data from the sensor, and auxiliary data from the power and ADC modules.

- Steim Compression and SDRAM Support: ShakeNet data collection uses the Tenet hierarchical architecture (Gnawali and others, 2006). We have a higher tier of master nodes that task the lower-tier nodes and collect the data responses coming from them. However, total data collection capability is capped by the limited wireless radio bandwidth. To overcome this limitation, we implemented the Steim2 algorithm (SEED Reference Manual, 1993) for compressing data aboard each ShakeBox prior to sending it to the master. In addition, wireless links are well known for their intermittent behavior. To better adapt to such conditions, we added 32 MB SDRAM support in the ShakeBox so that up to 14 hours of data (at $100 \mathrm{sps}$ ) can be buffered temporarily and then sent out whenever the link quality improves.

- GPS Time Sync: Time synchronization is required for correlation of data collected across multiple ShakeBoxes. Since ShakeBoxes will be placed inside a building or other structure with limited or no access to the open sky, using GPS for continuous time synchronization is not feasible. We developed a method to time-synchronize the mote and master cloud over USB but found it was not adequate, owing to the requirement of only a few millisecond error margin for time synchronization imposed by ANSS Class A requirements. The millisecond accuracy requirement also sometimes made using Flooding Time Synchronization Protocol (FTSP) 
infeasible because we were not able to estimate clock drift between master tier and sensor motes with millisecond accuracy. To overcome this, we alternatively use GPS to record the value of each ShakeBox's internal clock at the beginning and end of data collection. This assumes linear drift between the two recording times, which may not always true. Before deployment to the site we capture the sensor mote's internal clock against the GPS time under open sky. This is repeated after the end of the deployment but before turning off the ShakeBoxes. The two GPS time stamps at the start and end are used to calculate the drift in internal clock. We compensated the recorded internal clock time values during postprocessing of the data.

\section{ShakeNet Hardware: ShakeBox}

In collaboration with RefTek, we adopted a modular design for the ShakeBox (fig. $3 A, B$ ), which consists of four independent modules: CPU, power, analog-to-digital (ADC), and sensor, connected via standard $\mathrm{S}$ bus. Figure $3 C$ shows the $\mathrm{CPU}$, power, and $\mathrm{ADC}$ modules. These modules are housed in a custom-made weatherproof casing used in a number of RefTek instruments as shown in figure $3 \mathrm{~A}$.
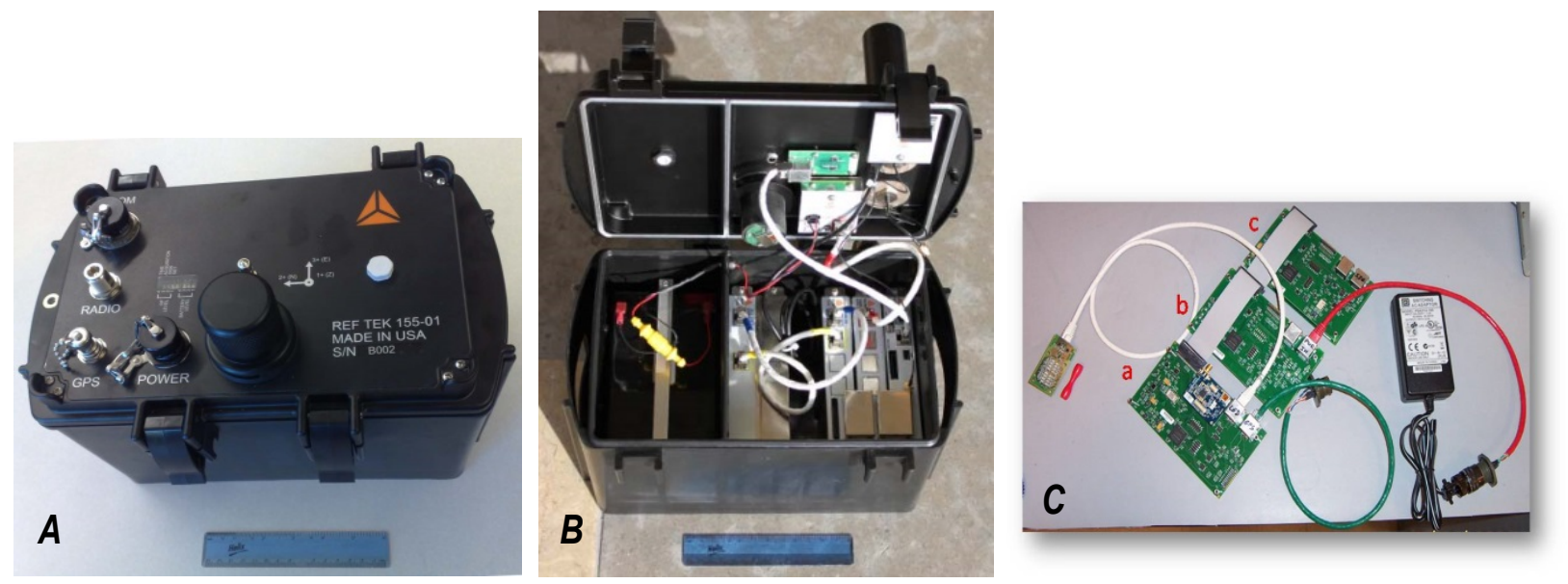

Figure 3. Photographs of the ShakeBox components. $A$, ShakeBox with weatherproof enclosure; six-inch ruler shown for scale. $B$, Placement of modules with 6-inch ruler shown for scale. $C$, Connection between individual modules: a, Central Processing Unit (CPU) module with Imote2; b, power module; c, Analog-to-Digital Converter (ADC) module.

The CPU module contains the system processor (a Crossbow Imote2) and the RT617 board; it controls all system operations. The Imote 2 controls communication with the other three modules via two SPI interfaces. The RT617 board consists of an FPGA, precision oscillator, battery-backed real-time clock, SD memory card slot, Global Positioning System (GPS) interface, and a board ID EEPROM. It also provides the timing for the power and ADC modules. The Imote2 is an advanced sensor network platform and consists of a PXA271A 32-bit microcontroller and CC2420 radio. It has multiple communication interfaces; prominent among those are the SPI, USB host and USB slave, JTAG (Joint Test Action Group), and AC97 audio codec. The CC2420 is an 802.15.4-compliant 2.4 GHz (gigahertz) radio, which can produce up to $256 \mathrm{Kbps}$ data rate. Dynamic scaling of the core frequency of the PAX271 microcontroller, ranging from $13 \mathrm{MHz}$ (megahertz) to $208 \mathrm{MHz}$, provides a varied range of options for balancing processing power with energy usage.

The power module provides the power requirements of the different components, and consists of the RT618 FPGA board and RT620 power board. The RT618 provides communication with the CPU 
module, an internal clock, control of the voltage monitor ADC converter, control of analog power supplies, and board ID EEPROM. The RT620 provides an input power controller, switching supplies at different voltage levels, a 16-bit ADC monitor for supply voltages and input currents, and a board ID EEPROM.

The analog-to-digital module takes the analog sensor inputs and provides a time-stamped 24-bit digital output. It consists of the RT618 FPGA board and RT614 analog board. The RT618 provides communication with the CPU module, a clock for time stamping sampling data, control of the ADC chips, test-signal generator for debugging, relay control, a board ID EEPROM, and sensor ID interface. The RT614 provides scaling of sensor signal voltages, three 24-bit ADC converters, replays to connect test signals to internal analog inputs, a board ID EEPROM, and voltage regulators.

The sensor module consists of three Colibyrs SiFlex 1500 accelerometers, which are interfaced to the RT614 board in the ADC module. The SiFlex 1500 operates from a bipolar power supply voltage that can range from \pm 6 to $\pm 15 \mathrm{~V}$ (volts) with a typical current consumption of $12 \mathrm{~mA}$ (milliamps) at \pm 6 $\mathrm{V}$. The linear full acceleration range is $\pm 3 \mathrm{~g}$ with a corresponding sensitivity of $1.2 \mathrm{~V} / \mathrm{g}$.

The weatherproof casing houses all the modules. Each module is electronically shielded to protect against electromagnetic disturbance. The lead acid battery used in the ShakeBox is placed in a separate sealed compartment to isolate it from the electronics in case of battery leakage. The box provides serial connectors, connector for GPS, LEDs (Light Emitting Diodes) for display and feedback, and antenna connector for a high gain external antenna used by the Imote2's radio. The instrument case has three screws and a spirit level for leveling. The prototype box shown in figure $3 A$ is made of a strong thermoplastic resin plastic.

Communication between modules in the ShakeBox is achieved with three buses: the module selection (SEL) bus whose purpose is to enable specific modules, the SPI command and control bus, and the AD data bus. While the SEL bus is used by the Imote 2 to select a specific component in a module, the SPI command and control bus (the SPI1 port on the Imote2) is used to communicate with that component. The AD data bus is used for the Imote 2 to collect data from the ADC module, along with auxiliary data from the power module. During development, we required debugging features to upload driver code and FPGA images to the boards, and to perform diagnostics. Thus, the board modules exposed the JTAG port for FPGA programming, and the Imote 2 was programmed and debugged using the USB slave port.

\section{Laboratory Testing and Experiments}

\section{FFT (Fast Fourier Transform) and Channel Analysis With Function Generator}

We conducted a series of experiments to test the accuracy of the ADC in the ShakeBox. The goal of the laboratory testing was to assess the fidelity and integrity of the various sensing hardware components. The equipment we used included an HP33120A Function Generator which accepts user parameters to generate an analog wave signal. We fed the input signal to the ShakeBox, collected the response from the ADC, and performed numerical analyses such as FFT (Fast Fourier Transform), and coherence and cross correlation between channels. We varied the frequency range from $0.2-125 \mathrm{~Hz}$, which covers most frequencies of interest in earthquake engineering analysis, and the amplitude range from 1-19 Vpp (volts peak-to-peak). We also measured the noise characteristics of each ADC channel, with and without the function generator connected. The tests were conducted with and without the sensor, at 125, 250, and $500 \mathrm{sps}$. The tests were also conducted comparing battery to AC power. With the sensor attached, tilt tests were conducted to measure response. Because of space limitations, we only 
present one set of results here-a sine-wave frequency sweep from 0.2 to $125 \mathrm{~Hz}$ with $1 \mathrm{Vpp}$ amplitude (fig. 4).

Figure 4 shows the FFT analysis for a sine-wave frequency sweep from 0.2 to $125 \mathrm{~Hz}$. It is readily apparent that the $\mathrm{ADC}$ has a reasonable response up to about $100 \mathrm{~Hz}$, and then the response drops dramatically from 100 to $125 \mathrm{~Hz}$. This is expected behavior according to the ADC datasheet.
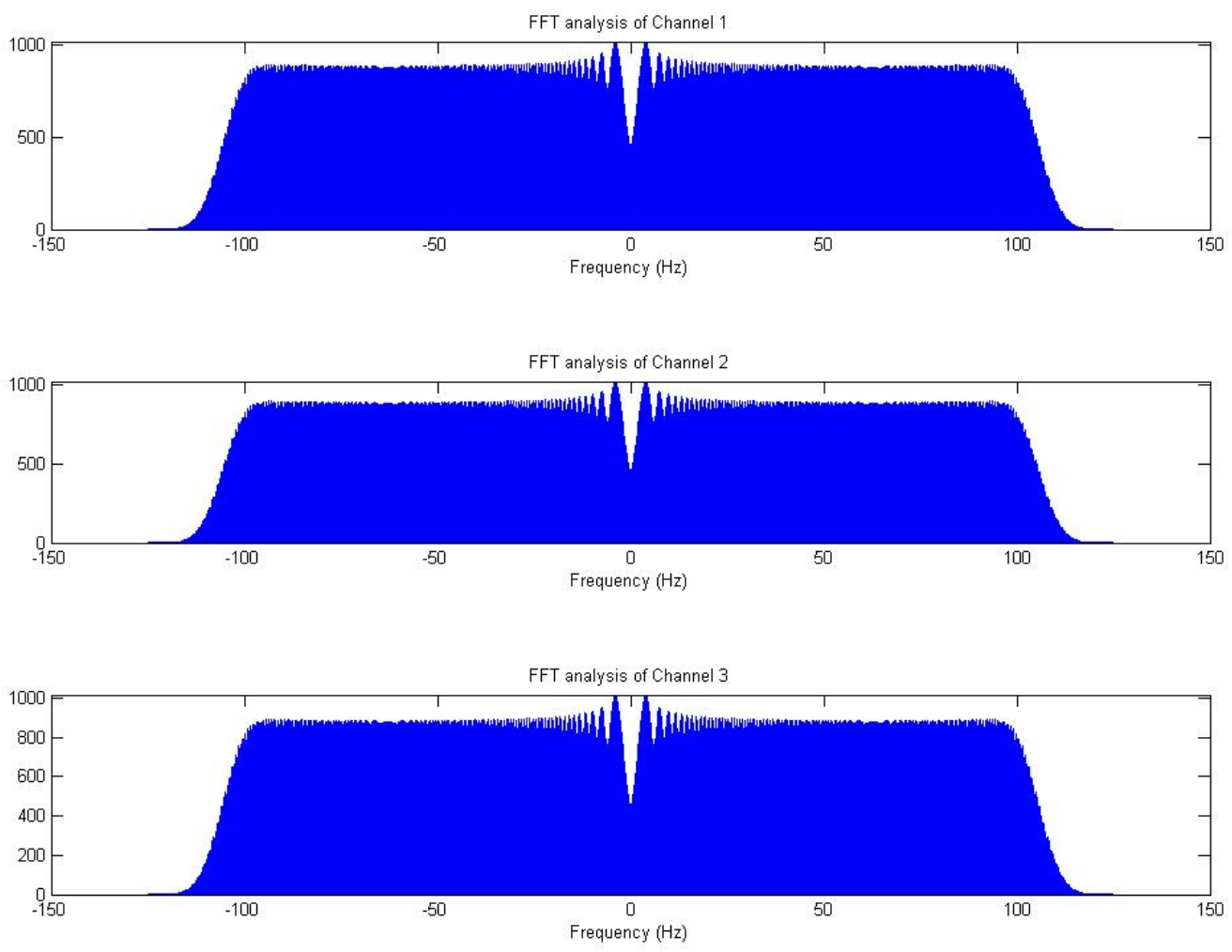

Figure 4. Graphs showing FFT (Fast Fourier Transform) analysis of input function generator frequency sweep from 0.2 to $125 \mathrm{~Hz}$ (hertz) with $1 \mathrm{Vpp}$ (volt peak-to-peak) amplitude. Negative frequencies are also plotted by default.

\section{Shake Table Test}

We conducted a unidirectional electrodynamic shake table test of the ShakeBox in the Department of Mechanical and Civil Engineering at Caltech. The shake table input was fixed at frequencies between 0.1 and $90.0 \mathrm{~Hz}$, and the ShakeBox response was compared with collocated Dytran piezoelectric accelerometers. Figure 5 shows the shake table test equipment and setup. Halfway through the test, the sensors were rotated to test both orthogonal horizontal directions.

We ran the unidirectional shaker at $0.1,0.5,0.8,1.0,1.2,1.5,2.0,5.0,10.0,25.0,49.0,50.0$, and $90.0 \mathrm{~Hz}$ for between 10 seconds and two minutes using a sinusoidal wave as input. A 2-minute ambient vibration test was conducted at the end of the test. Nine Dytran piezoelectric accelerometers were attached to the sides and top of a mounting plate (three in each direction). These were connected to a 24bit Granite digitizer with GPS antenna. The Dytran accelerometers have flat response for 1-2,000 Hz with a range of $\pm 50 \mathrm{~g}$ and were calibrated at the factory in early 2009. During the tests, we compared the 
Dytran-Granite versus ShakeBox performance in near-real time and observed strong similarities in waveform shape and amplitudes. Though the input was a sine wave, the shaker appeared to give a little kick at each end of its travel distance. This kick produced additional peaks in the output seen on both the Dytran and Colibrys sensors. Because we observed the response to these kicks on both sets of accelerometers, we concluded that it was not caused by a fault in accelerometer response. Even though the channels were lined up parallel to and perpendicular to the direction of shaker motion (fig. 5, $A$ and $B$ ), the motion was not perfectly one-dimensional; a response was also observed in the other orthogonal channels but at lower amplitudes. This is partly due to shake table imperfections, but it may also be due to cross-axis misalignments between the sensors' indicated and true axes. Dytran-Granite timing was based on GPS time stamps, and the ShakeBox timing was based on time stamps from the PC clock. The Dytran-Granite GPS time stamps were used as the reference start times.
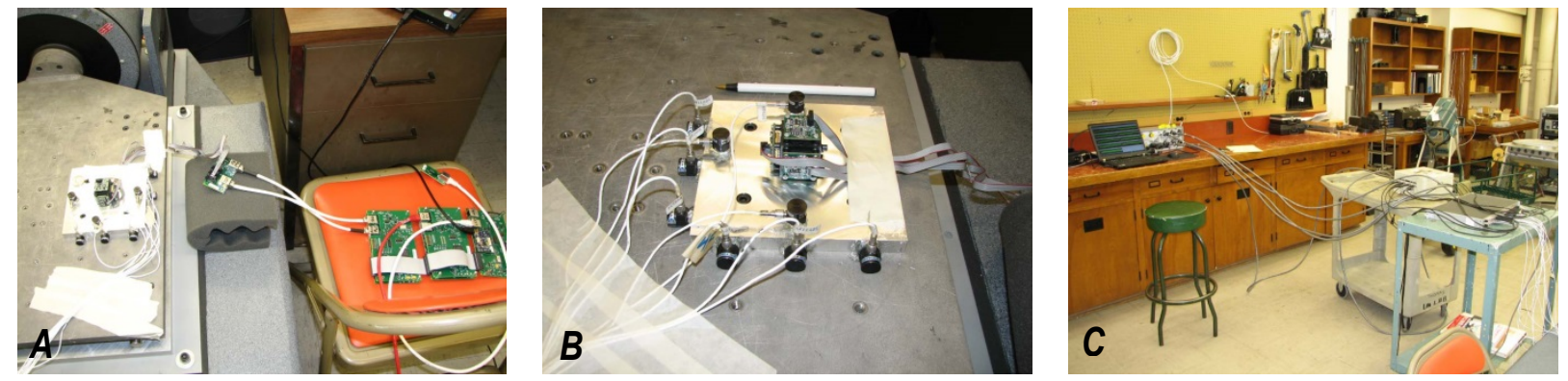

Figure 5. Photographs showing shake table equipment and instrumentation setup. $A$, Shake table top showing mounted steel plate with Dytran piezoelectric and Colibrys MEMS accelerometers, and additional ShakeBox components on chair. B, Closeup of mounted plate with accelerometers. C, 24-bit digitizer and PC recording setup for Dytran accelerometers.

For each frequency, we compared waveform shapes between Dytran-Granite and ShakeBox performance, expecting differences to be indications of questionable or faulty performance in the Colibrys. Examination of absolute amplitudes showed that they were within 10 percent or better of each other (fig. 6). The observed spectral peaks in the FFTs computed from the acceleration time series of both types of sensors also showed good agreement between the two systems. 
A Shake table at $1.0 \mathrm{~Hz}$ in MEMS Ch. 2 direction
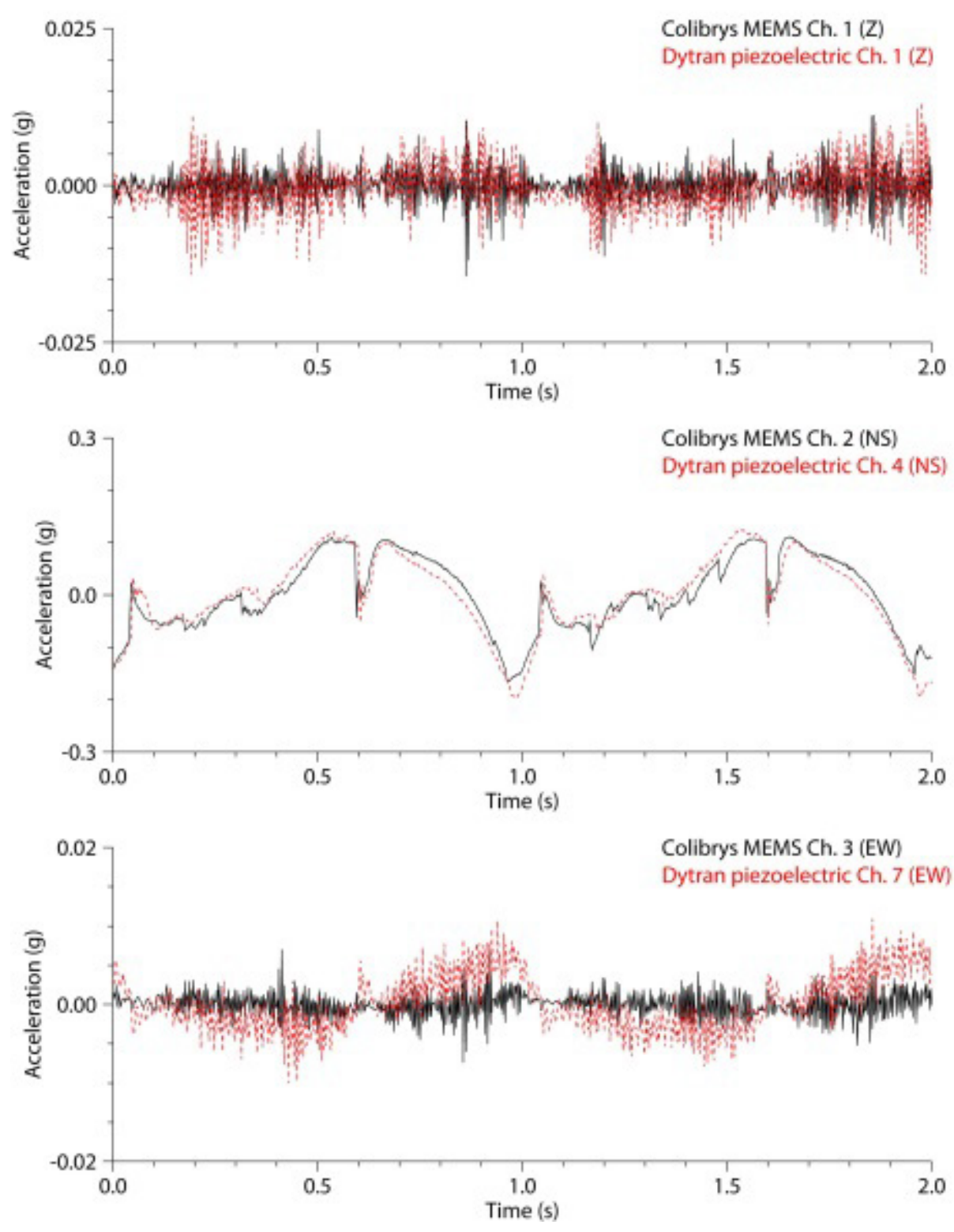

Figure 6. A, Shake table test results comparing ShakeBox (Colibrys MEMS: black curves) with Dytran piezoelectric accelerometer response (red curves), recorded at $100 \mathrm{sps}$. Shaking was performed in the Colibrys Channel 2 direction with $1 \mathrm{~Hz}$ sine wave input. 
B

Shake table at $5.0 \mathrm{~Hz}$ in MEMS Ch. 2 direction
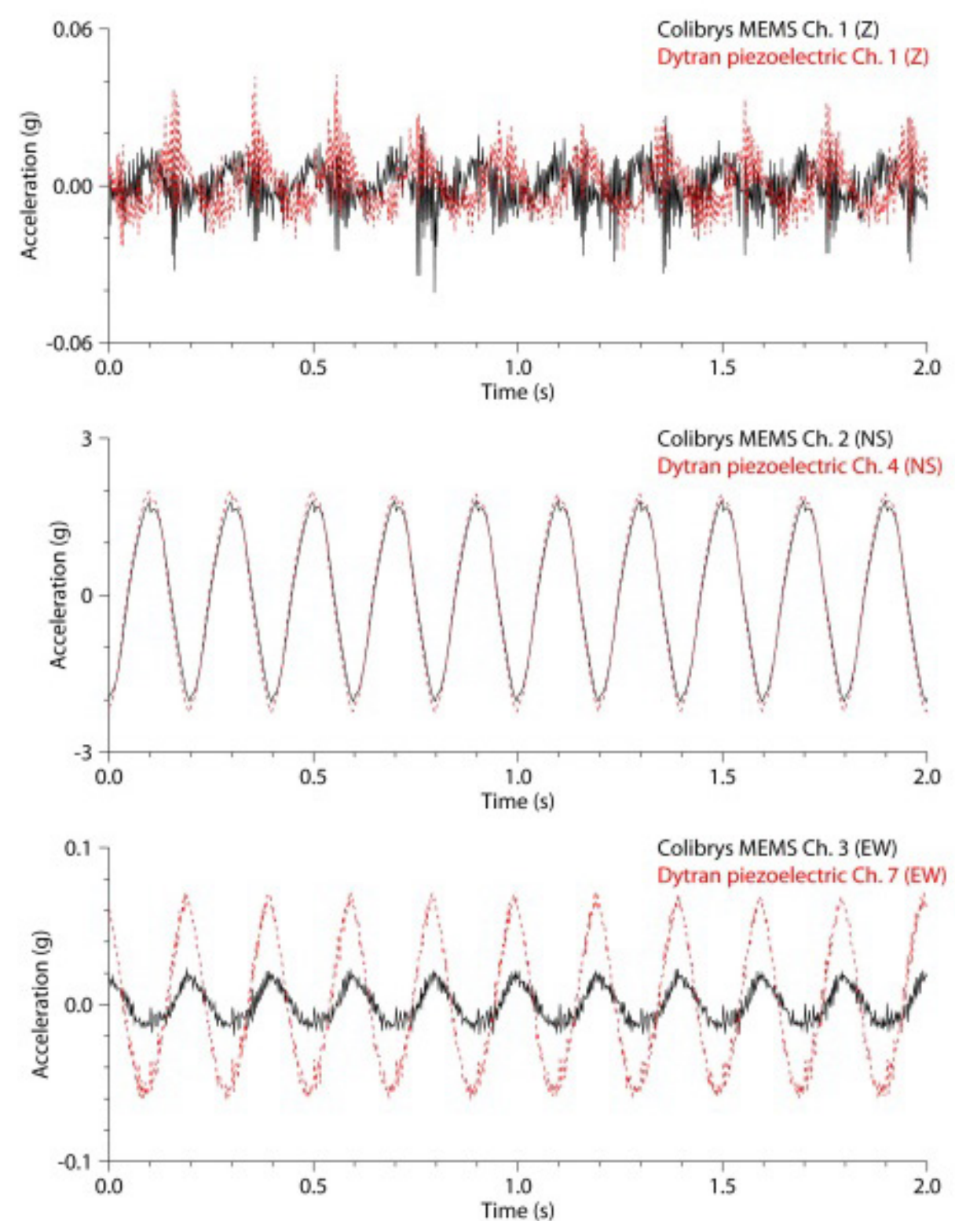

Figure 6.-Continued B, Shake table test results comparing ShakeBox (Colibrys MEMS: black curves) with Dytran piezoelectric accelerometer response (red curves), recorded at $100 \mathrm{sps}$. Shaking was performed in the Colibrys Channel 2 direction with $5 \mathrm{~Hz}$ sine wave input. 

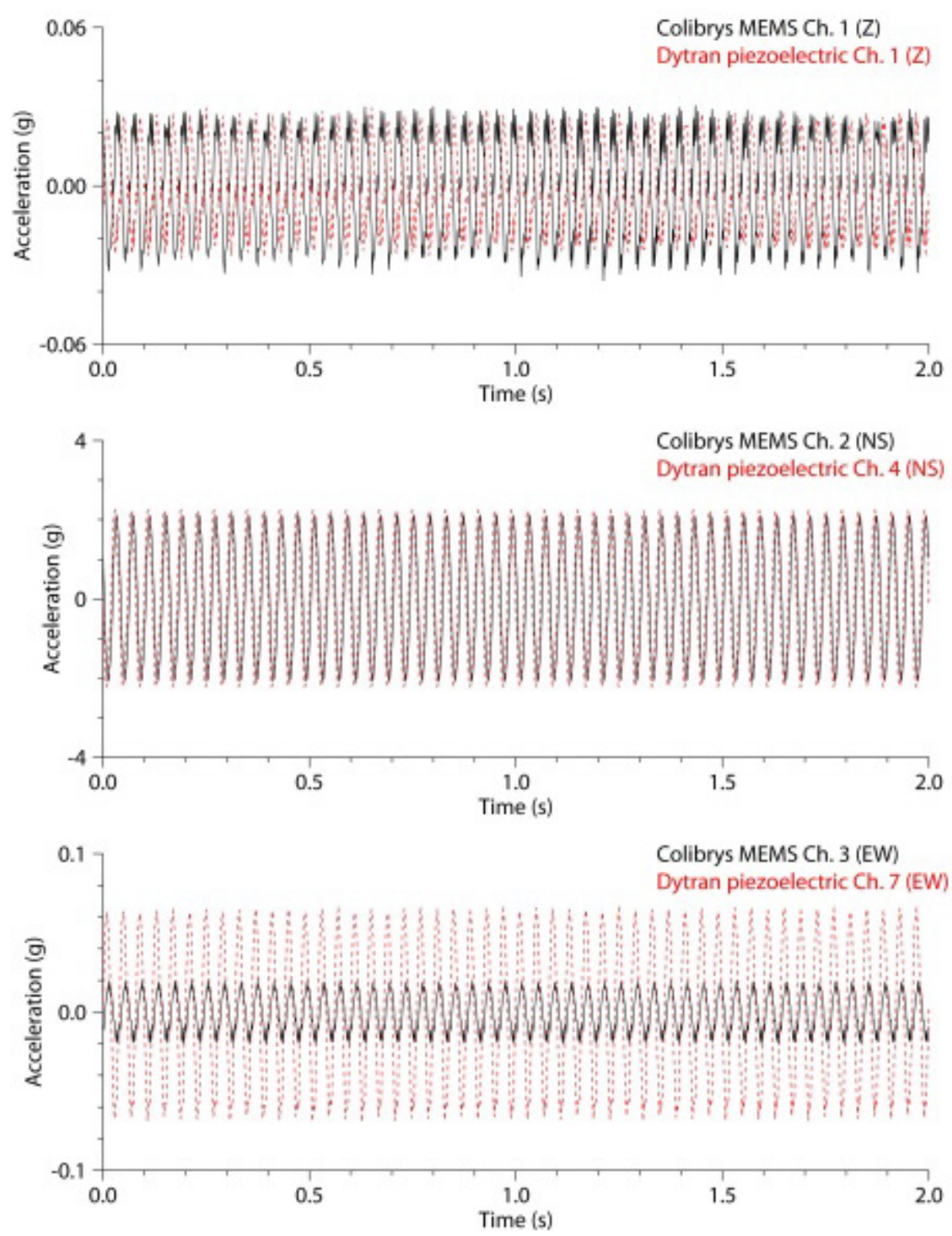

Figure 6.-Continued C, Shake table test results comparing ShakeBox (Colibrys MEMS: black curves) with Dytran piezoelectric accelerometer response (red curves), recorded at $100 \mathrm{sps}$. Shaking was performed in the Colibrys Channel 2 direction with $25 \mathrm{~Hz}$ sine wave input.

\section{Millikan Library Test}

We conducted a ShakeBox performance test at Millikan Library on the Caltech campus (fig. 7). The objective was to compare the ShakeBox performance versus the permanent Episensor accelerometers' performance during several forced vibration tests. The Episensor is a broadband forcebalance accelerometer used in permanent, wired, structural arrays and has reliable, low-noise 
performance. An eccentric-mass shaker is located on the Millikan Library roof and provided the excitation in a single horizontal direction during any one shaking test (fig. 7). Although the building responds in other axes as well, our comparison of collocated sensors obviates any issues arising from such responses and are useful, as described below. Two Shakeboxes were collocated with threecomponent Episensor accelerometers on the $9^{\text {th }}$ floor and in the basement (fig. 7). Millikan Library is a nine-story reinforced concrete building consisting of both shear walls and moment frames. Shear walls dominate the east and west faces of the building and also line the elevator core in the center of the building. The shaker was run at frequencies between 1.0 and $9.5 \mathrm{~Hz}$, and each ShakeBox response was compared with the collocated Episensor accelerometers.

The forced vibration tests consisted of an initial empty-buckets test (no weights added resulting in minimum applied force), a full-buckets test (all weights added resulting in maximum applied force) in the east-west direction, and a full-buckets test in the north-south direction. The empty-buckets shake spanned frequencies from 1.0 to $9.5 \mathrm{~Hz}$ gradually over the course of approximately 40 minutes. The rate changed over the course of the sweep. The lowest frequencies progressed at $0.05-\mathrm{Hz}$ intervals every $20 \mathrm{~s}$ (seconds), from 2.5 to $5 \mathrm{~Hz}$ progressing at $0.05 \mathrm{~Hz}$ intervals every $15 \mathrm{~s}$, and from 5 to $9.5 \mathrm{~Hz}$ progressing at $0.05 \mathrm{~Hz}$ intervals every $10 \mathrm{~s}$. This frequency range covers the building's first east-west mode frequency of $1.2 \mathrm{~Hz}$ (ambient), the first north-south mode frequency of $1.7 \mathrm{~Hz}$ (ambient), and the first torsional mode frequency of $2.4 \mathrm{~Hz}$ (ambient). The second test consisted of a full-buckets shake (full set of weights for increased force, resulting in temporary nonlinear building response) for frequencies between 1.0 and $2.5 \mathrm{~Hz}$, particularly to excite the first east-west frequency at $1.15 \mathrm{~Hz}$ (nonlinear response due to maximum force applied). The sweep up to $2.5 \mathrm{~Hz}$ and back down to the starting frequency of $1.0 \mathrm{~Hz}$ took approximately 30 minutes. The east-west full-buckets test also excited the torsional mode $(2.3 \mathrm{~Hz}$, nonlinear) in a narrow band around the mode progressing at a rate of $0.2 \mathrm{~Hz}$ intervals every $45 \mathrm{~s}$. At the end of this test, we paused near the peak resonance for around a minute at both the first east-west and torsional mode frequencies.

The first east-west frequency shake shows up primarily in the east-west components of the accelerometers, and the first torsional shake shows up clearly in both the east-west and north-south components. The third test consisted of a full-buckets frequency sweep in the north-south direction for frequencies between 1.0 and $1.8 \mathrm{~Hz}$, particularly to excite the first north-south frequency at $1.6 \mathrm{~Hz}$ (nonlinear). The north-south full-buckets test excited the first north-south frequency in a narrow band around the mode progressing at a rate of $0.2 \mathrm{~Hz}$ intervals every $45 \mathrm{~s}$. At the end of the test, we paused again at resonance on our way back to $0 \mathrm{~Hz}$. As the shaker frequency intersected these modes, we observed increased response resulting in increased amplitudes.

The recorded accelerations for both types of instruments in the basement and $9^{\text {th }}$ floor locations are shown in figure 8, plotted in the same units $(\mathrm{mg})$ (milli-g where $g$ is units of gravitational acceleration). Note that the amplitude scales are different for each component. Overall, the response of the ShakeBox (Colibrys) is quite similar to that of the Episensor-Granite except that the ShakeBox amplitudes are usually about 10-20 percent larger. This may be due to the lack of robust physical coupling of the ShakeBox with the floor slab in both locations (we placed our boxes on the floor, whereas the Episensors are bolted into floor slabs or walls). The amplitude difference is larger at the $9^{\text {th }}$ floor where the shaking was stronger than in the basement. 

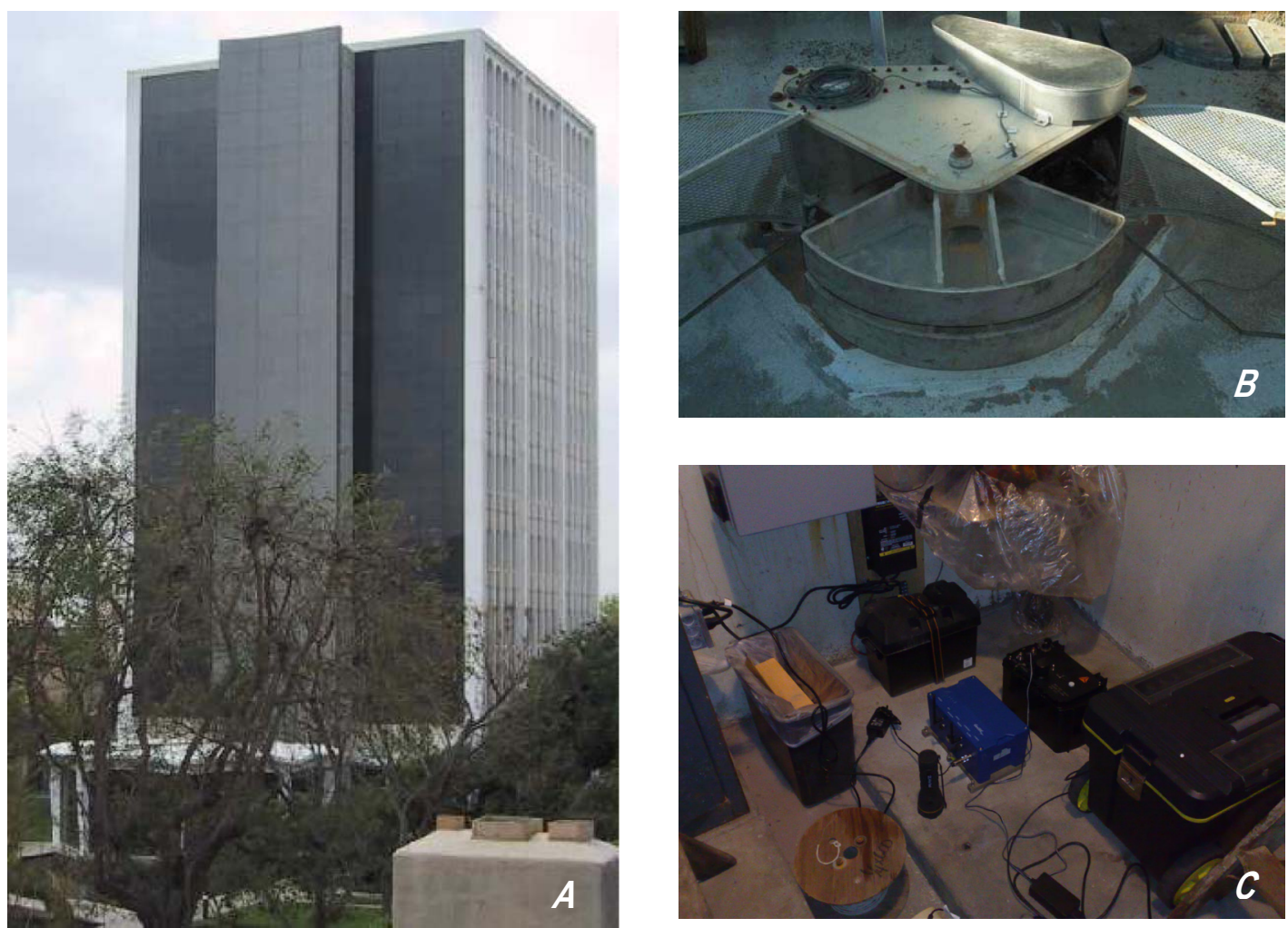

Figure 7. Photographs showing Robert A. Millikan Memorial Library, shaker, and sensors that were tested. $A$, Robert A. Millikan Memorial Library on the Caltech campus, viewed from the northeast. $B$, Kinemetrics VG-1 eccentric-mass shaker located on the roof of Millikan Library. The counter-rotating buckets, shown empty, can be loaded with different configurations of lead weights to impart different forces on the building. C, Accelerometers located in the basement that were recording during the Millikan shake test. The ShakeBox is the small black box with the orange triangle symbol on top, behind the blue box. 

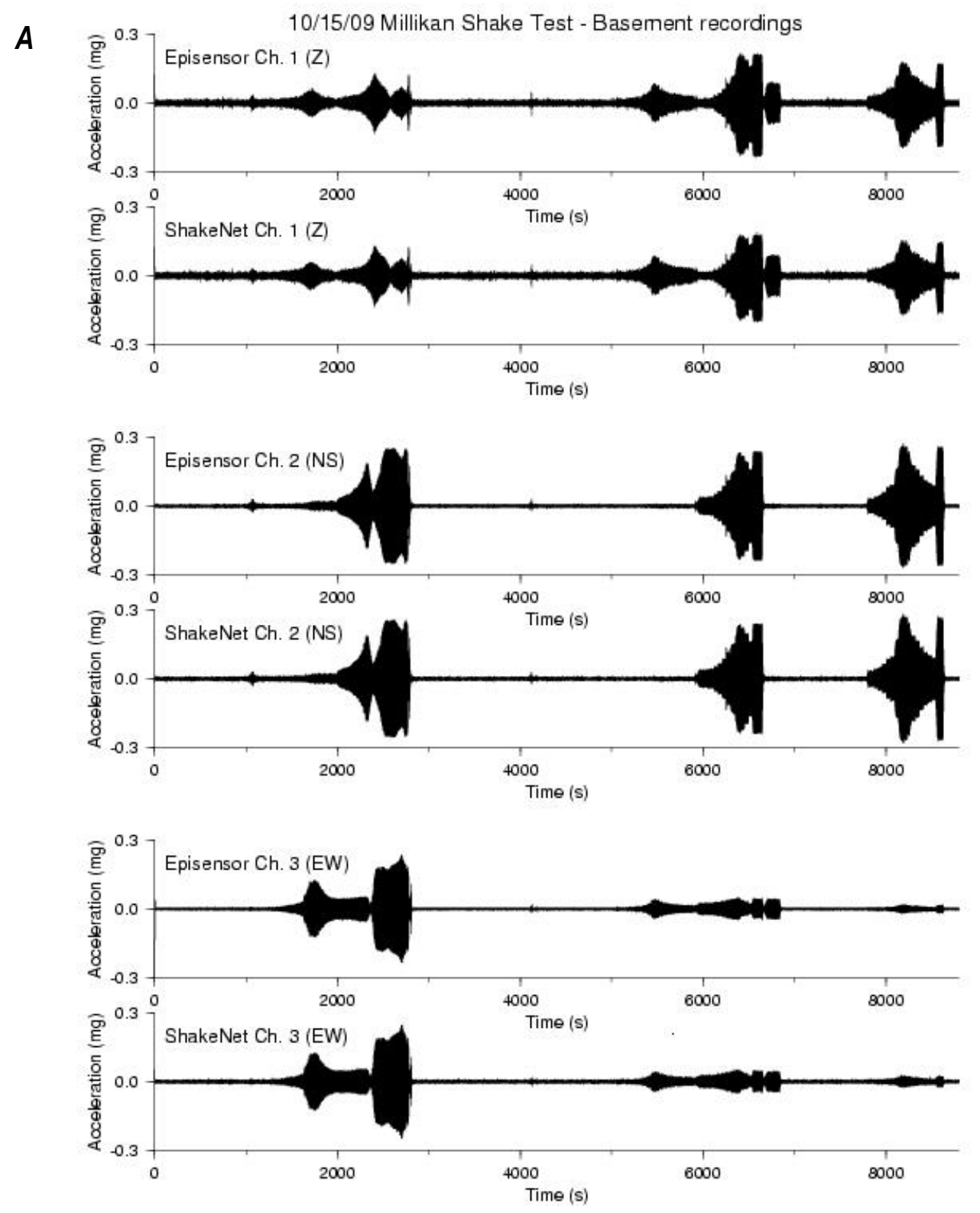

Figure 8. A, Graphs showing acceleration responses due to forced vibrations recorded by the ShakeNet ShakeBoxes collocated with an Episensor in the basement of Millikan Library, California Institute of Technology. 

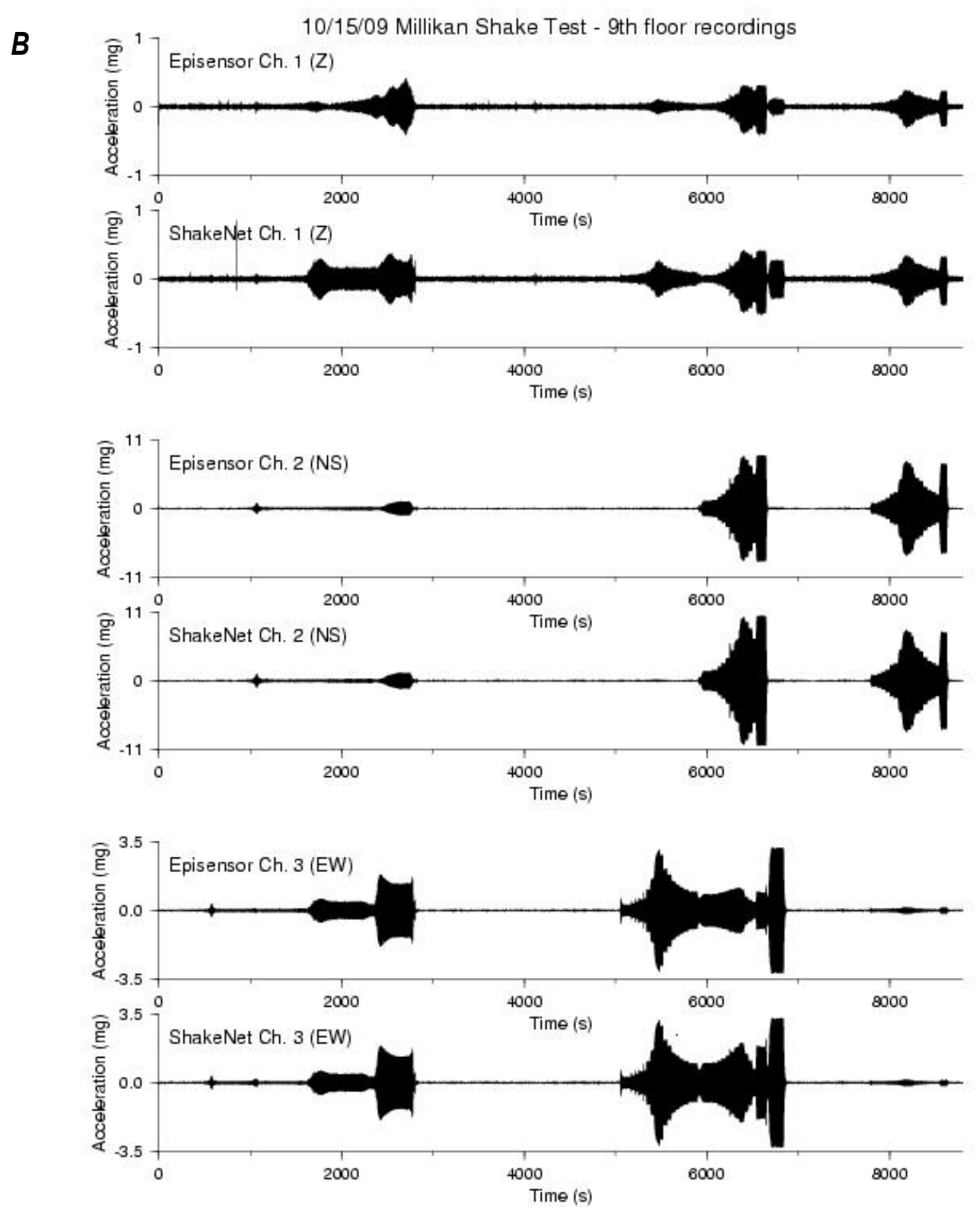

Figure 8.-Continued $B$, Graphs showing acceleration responses due to forced vibrations recorded by the ShakeBoxes collocated with an Episensor on the $9^{\text {th }}$ floor of Millikan Library, California Institute of Technology.

\section{Prototype Deployments in Structures}

\section{Deployment of Instruments During Early Development}

In 2006, coauthor Govindan's group deployed a 20-node, wireless, ShakeNet prototype network on the Vincent Thomas Bridge in San Pedro, Calif. (Paek and others, 2006) (fig. 9). One day of ambient vibration recordings from this deployment illustrates the ability to record coherent signals, most likely produced by passing truck traffic (fig. 10). Figure 10 shows the consistent spectra across sensors for this 
type of stand-alone deployment, exhibiting frequencies of bridge vibration that are in agreement with previous, longer deployments that recorded earthquake data with a traditional wired seismic array (Smyth and others, 2003).

The ShakeNet prototype was also tested on the steel, moment-frame, UCLA Factor building, which has an embedded, wired, 72-channel array. In 2006, we tested several motes on the $13^{\text {th }}-15^{\text {th }}$ and $1^{\text {st }}-3^{\text {rd }}$ floors, revealing the strengths and weakness of the instrument design, eventually leading to the current data acquisition board and MEMS accelerometer layout described earlier in this report.

\section{ShakeNet Prototype Deployments}

Our wireless ShakeNet strong-motion network was specifically designed to be deployed in structures with no existing power or communications infrastructure. We chose two test structures to collect information representing a range of environmental conditions. They included the building at 1100 Wilshire Boulevard in downtown Los Angeles, Calif., and the Seven Oaks Dam in Highland, Calif. The building at 1100 Wilshire Boulevard consists of a large, square-plan, 15-story, reinforced concrete base structure that holds a 700-space parking structure. A 21-story, steel moment-frame, triangular prismatic pentahedron sits on top of this base structure. The Seven Oaks Dam sits within 1 $\mathrm{km}$ of the San Andreas Fault and is located in a region of alluvial sediments in the southern foothills of the San Bernardino Mountains. It is a 550-ft-high, 2,980-ft-long (168 by $908 \mathrm{~m}$ ), earth-and-rock- fill dam designed to provide flood protection to Orange County, Calif. 
$A$

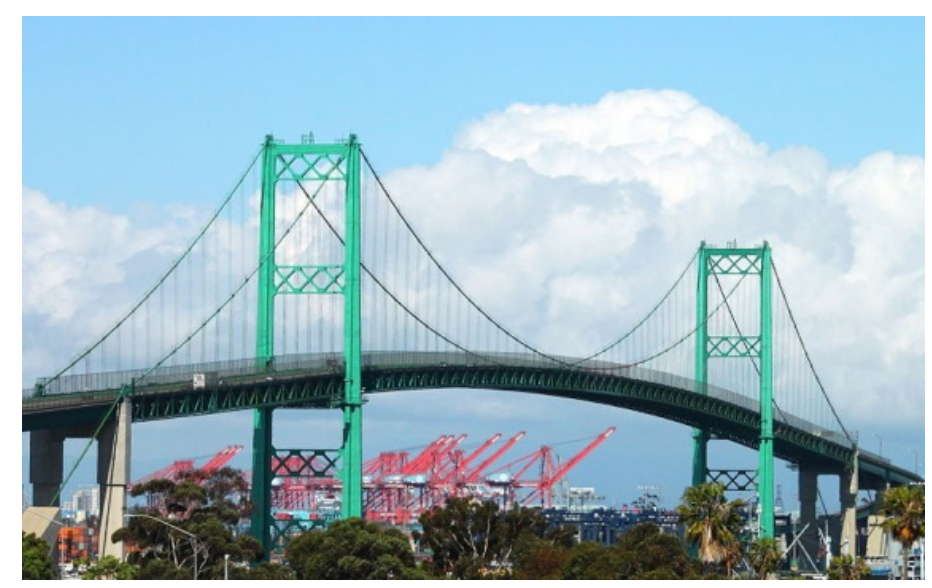

$B$

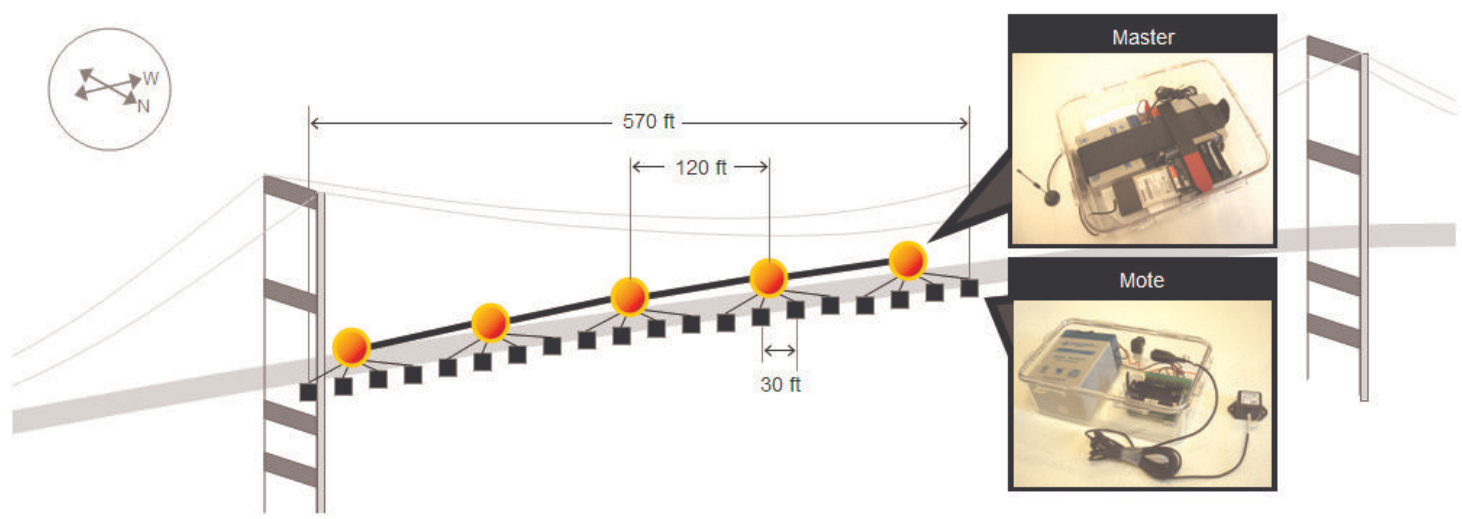

Figure 9. A, Vincent Thomas Bridge, Los Angeles Harbor, California. B, ShakeNet prototype deployment across part of the bridge deck. 


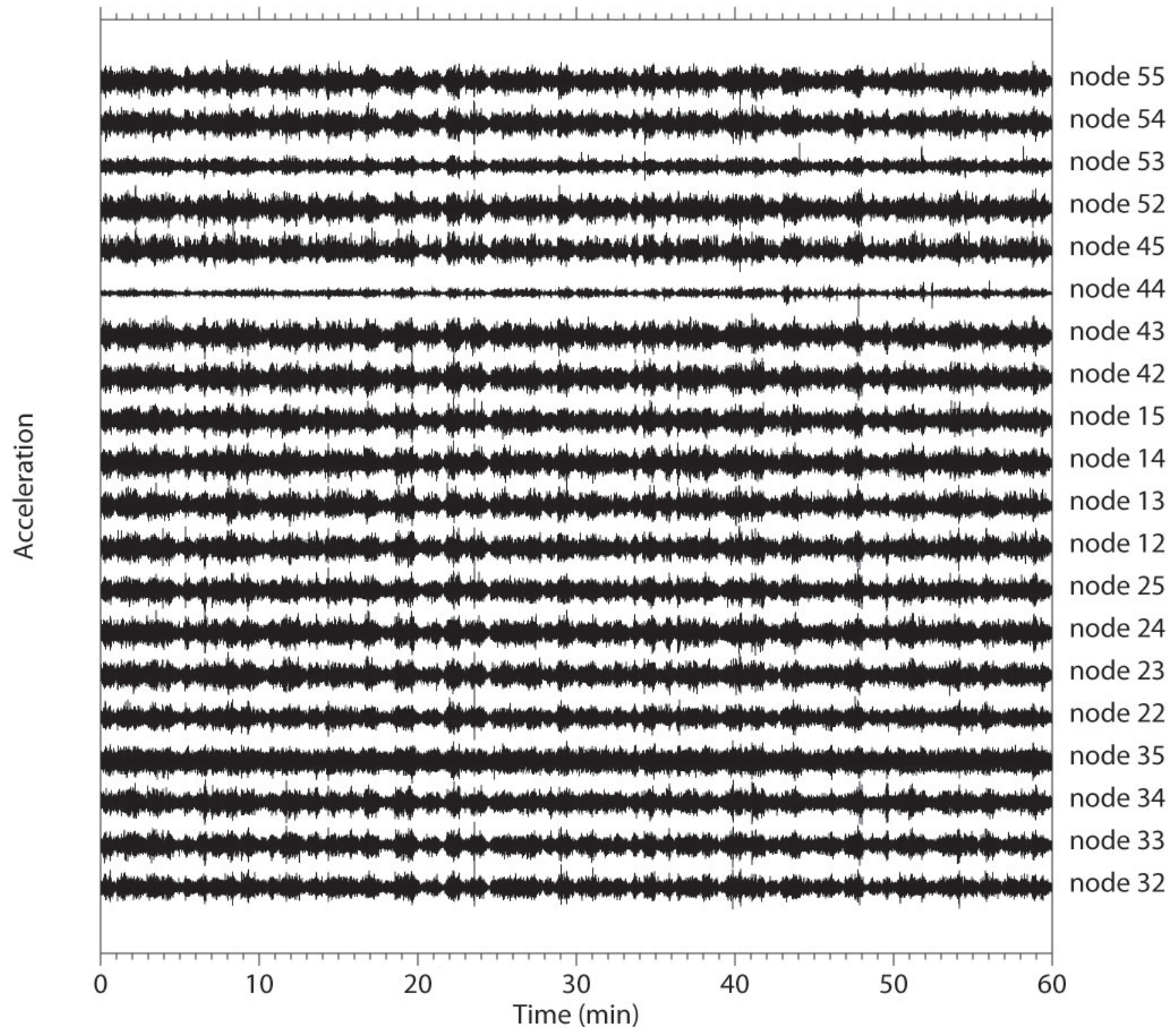

Figure 10. Graph showing accelerations recorded during the deployment at Vincent Thomas Bridge, Los Angeles, California, in July 2006. Vertical accelerations have been bandpass filtered for frequencies between 0.2 and $5.0 \mathrm{~Hz}$. 


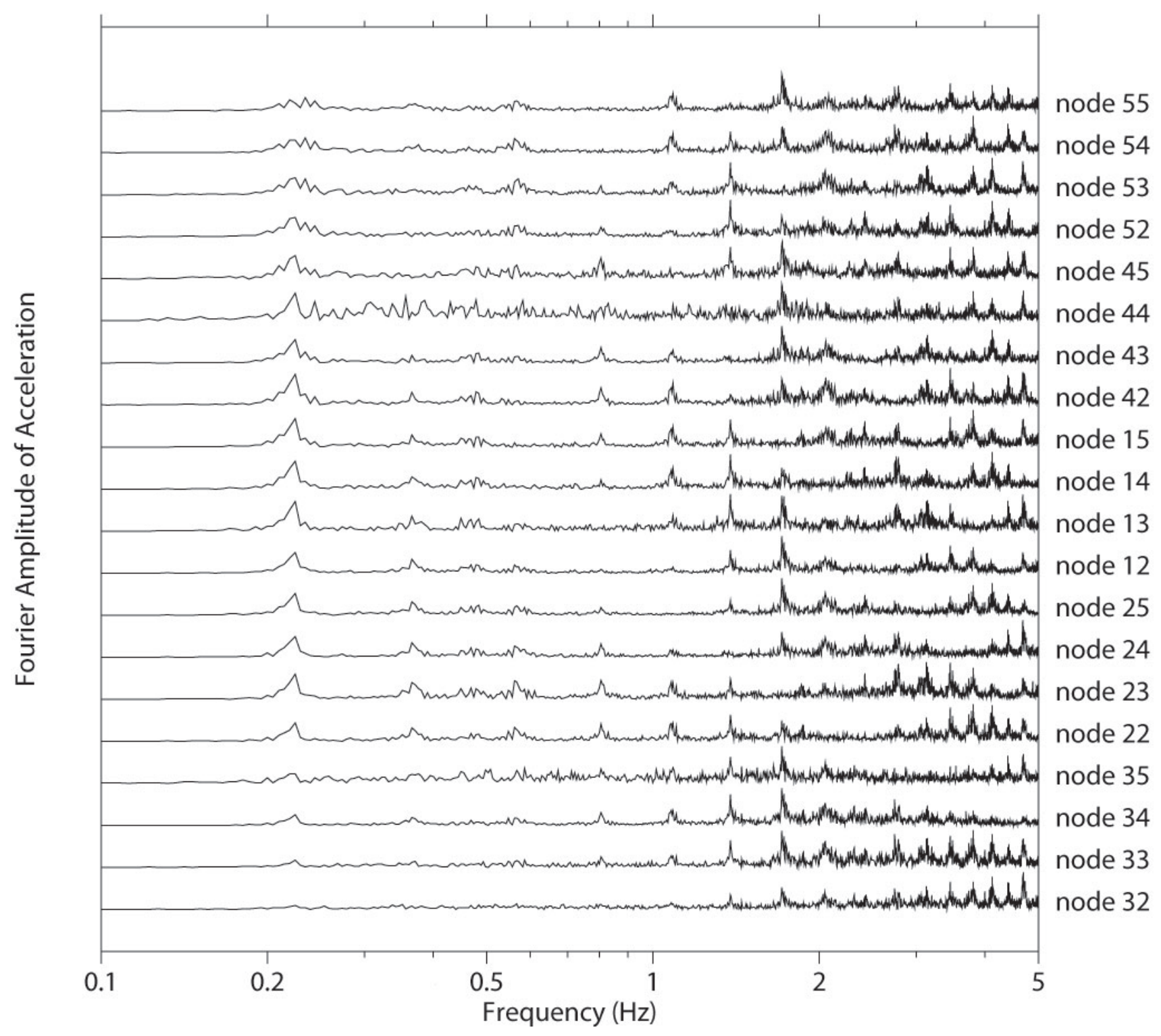

Figure 10.-Continued Graph showing spectra of accelerations recorded during the deployment at the Vincent Thomas Bridge, Los Angeles, California, in July 2006.

\section{Wilshire Boulevard Building}

In June 2011, we conducted our first ambient vibration deployment at the 1100 Wilshire Boulevard building. The 1100 Wilshire Boulevard building consists of a massive 15-story concrete cube that holds a 700 -space parking structure. The building is $483 \mathrm{ft}(147 \mathrm{~m})$ tall. A 21 -story steel momentframe, triangular prismatic pentahedron sits on top of the cube (fig. 11). 

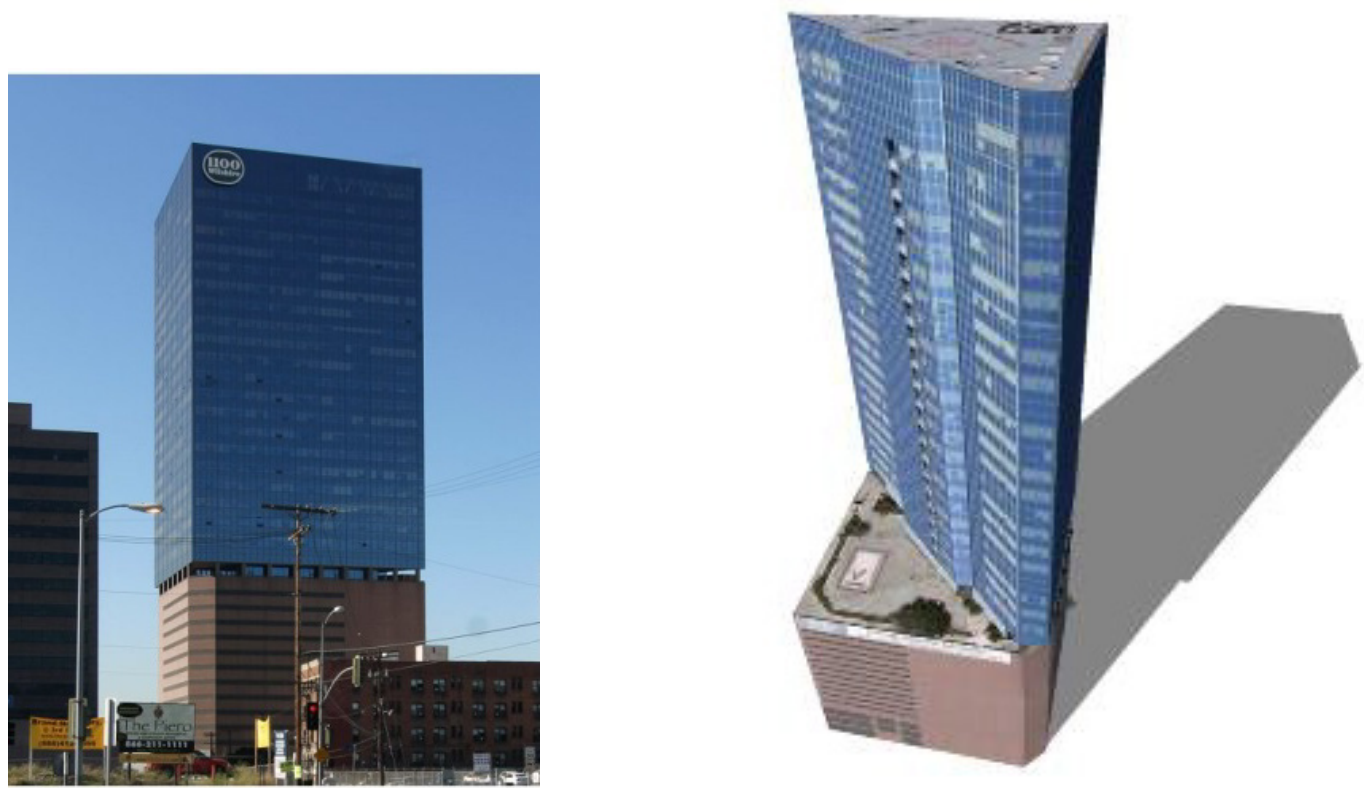

Figure 11. Photo and SketchUp ${ }^{\mathrm{TM}}$ diagram of the 1100 Wilshire Boulevard building in Los Angeles, California.

For three days, we operated 30 triaxial ShakeBoxes with 10 masters in the stairwells of several floors in the upper condominium section of the building, as well as along outer walls on several floors of the garage. The network recorded ambient vibrations of the structure for the 3-day time period. Because the seismometers used ultra-low power 802.15.4 radios, they did not interfere with local Wi-Fi communications. The network configuration is shown in table 1. 
Table 1. Floors with sensors in the 1100 Wilshire Boulevard building deployment, Los Angeles, California. [\#, total number]

\begin{tabular}{|l|l|l|}
\hline \multicolumn{2}{|c|}{ \# ShakeBoxes } & \# Masters \\
\hline B3 & 3 & 1 \\
\hline B & 3 & 1 \\
\hline 1 & 2 & 0 \\
\hline 2 & 2 & 2 \\
\hline 3 & 2 & 0 \\
\hline 16 & 2 & 0 \\
\hline 17 & 2 & 2 \\
\hline 18 & 2 & 0 \\
\hline 20 & 2 & 0 \\
\hline 21 & 2 & 2 \\
\hline 22 & 2 & 0 \\
\hline 36 & 2 & 0 \\
\hline 37 & 2 & 2 \\
\hline 38 & 2 & 0 \\
\hline
\end{tabular}

Based on a predeployment site reconnaissance visit, we found that wireless communication was excellent in the open stairwells, likely because there is a significant amount of metal in the numerous handrails, as well as the stairs and inside the walls, providing a waveguide effect through the otherwise open space. Even for floors that were tall, such as the lobby and conference room levels, communication from floor to floor within the stairwell was better. Although we expected that we might need to use multihop, placing the master in the middle of each cluster in nearly every case provided direct motemaster communication.

The primary weakness of this deployment turned out to be the masters' hardware. Some were not as reliable as expected and failed upon loss of communication. The eboxes used here had several issues. First, the operating system (Ubuntu 10.04) became unstable for a few specific CPU frequencies and the operating system would eventually hang. Second, not all USB ports functioned well. If the master mote was attached to a bad USB port, the data collection would stop halfway through, and the communication between the master and ShakeBoxes was lost. On the other hand, throughout the 1100 Wilshire Boulevard building deployment, we were able to evaluate the integration of the whole system and test the lifetimes of both ShakeBoxes and eboxes powered by car batteries under realistic deployment conditions.

Spectra from a section of horizontal records from acceleration data recorded in the building at 1100 Wilshire Boulevard are shown in figure 12. The spectra have been arranged in order of increasing height inside the building with the bottom six spectra from within the parking garage cube. The more flexible steel frame triangular prismatic pentahedron produces peaks in the spectra that are more obvious to identify than the stiffer reinforced concrete cube. The upper stories show distinguishable peaks at $0.25 \mathrm{~Hz}$ (possibly the first translational mode), $0.4 \mathrm{~Hz}$ (first torsional mode), $0.7 \mathrm{~Hz}$ (second torsional mode), $0.8 \mathrm{~Hz}, 0.9 \mathrm{~Hz}, 1.4 \mathrm{~Hz}, 1.6 \mathrm{~Hz}, 2.0 \mathrm{~Hz}$ and higher, also identified by Celebi and others (1991). The spectral lines in the spectrogram above $10 \mathrm{~Hz}$ are most likely due to machinery running at a 
range of frequencies and for limited time durations (for example, heating, ventilation, air conditioning, and elevators).

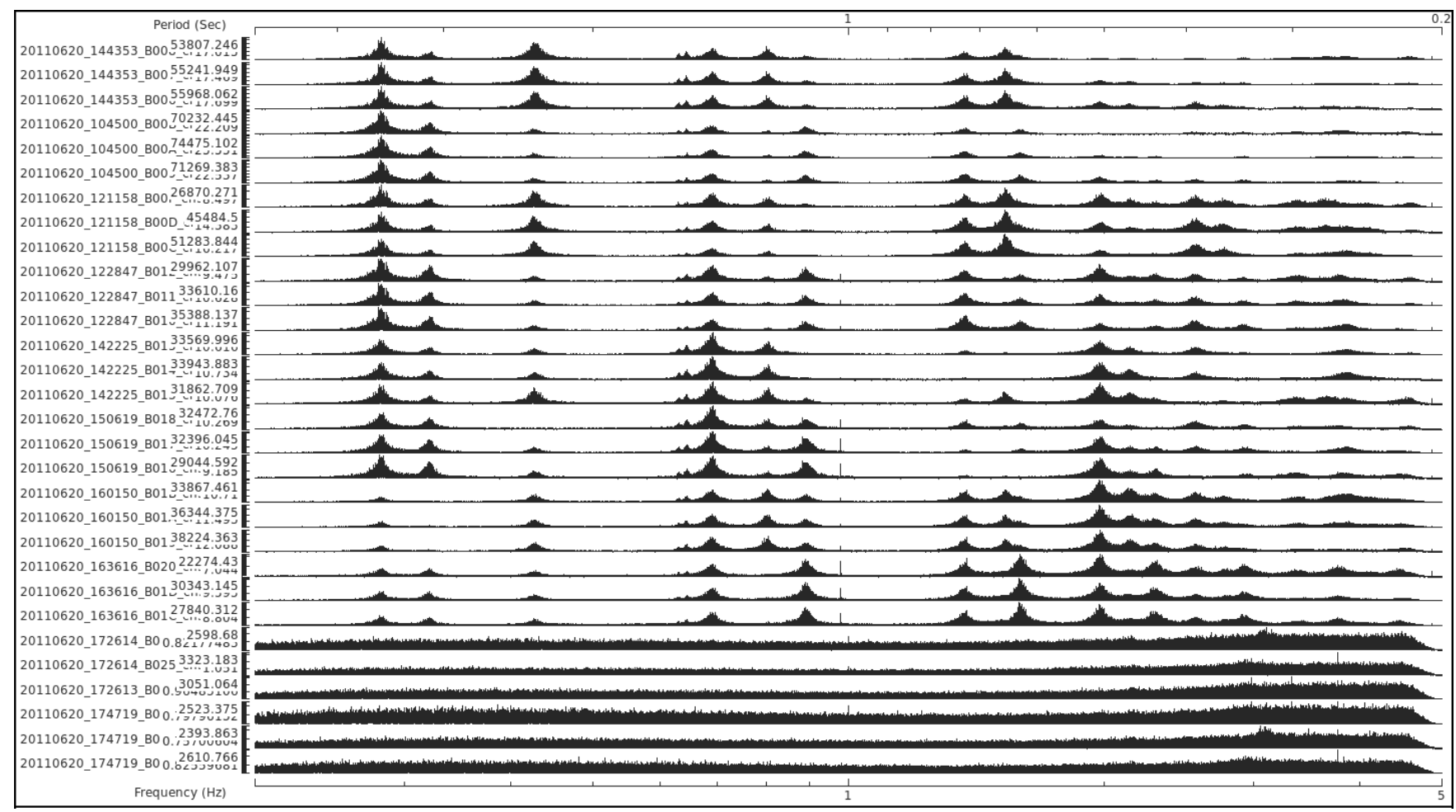

Figure 12. A, Spectra from a 1-hour section of horizontal component waveforms from 30 ShakeBoxes deployed in stairwells on various floors arranged from bottom to top of building (bottom to top of figure) at 1100 Wilshire Boulevard, Los Angeles, California. Numbers overwriting file name in left-hand column indicate amplitude normalization factor. 


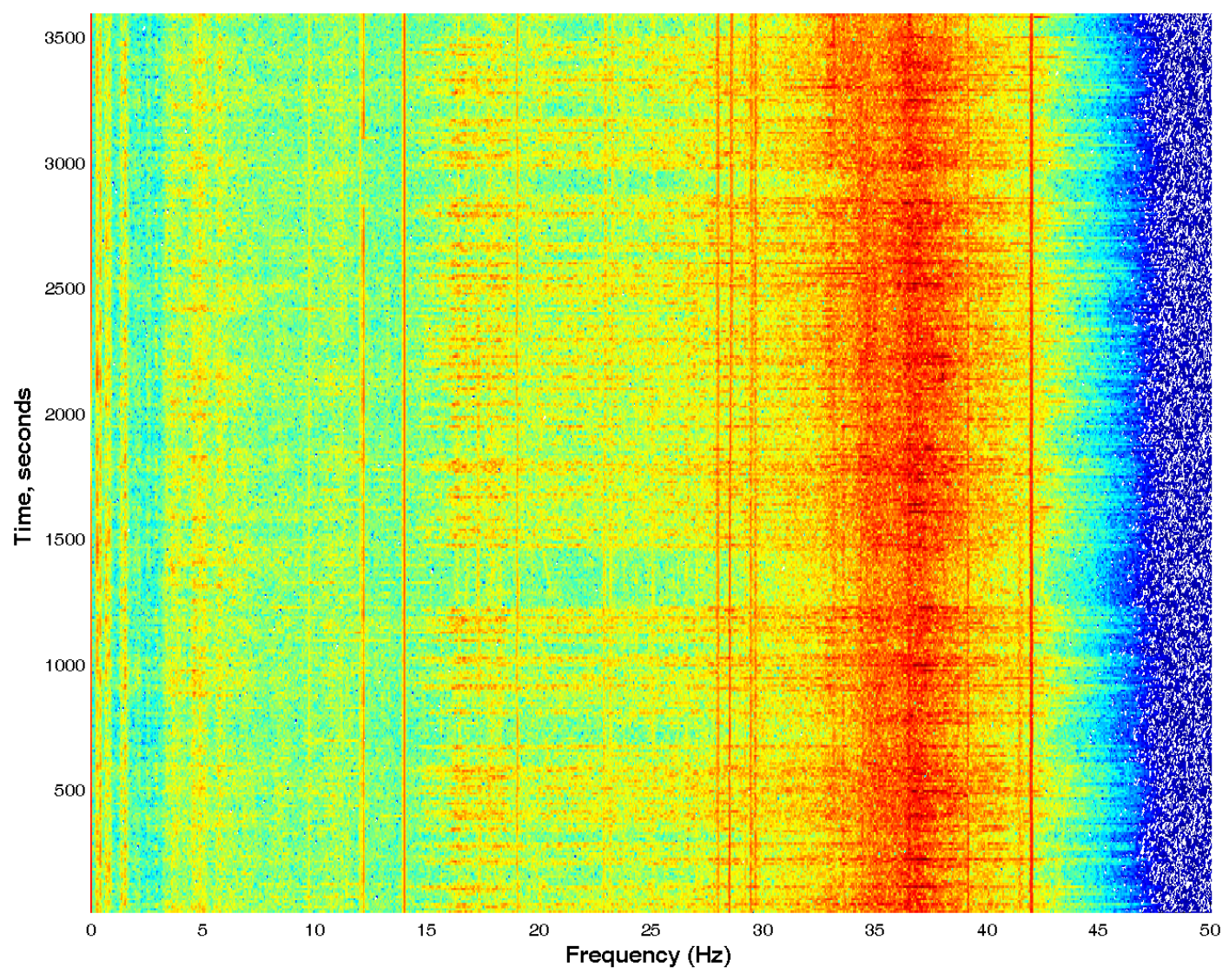

Figure 12.-Continued $B$, Spectrogram from a section of horizontal component data recorded by a ShakeBox deployed on the $36^{\text {th }}$ floor, from the test deployment in the building at 1100 Wilshire Boulevard, Los Angeles, California. 


\section{Seven Oaks Dam}

In October 2011, we conducted our second deployment at the Seven Oaks Dam (fig. 13). The Seven Oaks Dam sits within $1 \mathrm{~km}$ of the San Andreas Fault and is located in a region of alluvial sediments in the southern foothills of the San Bernardino Mountains. For three days, we set up and operated seismometers in 31 locations along the Seven Oaks Dam crest road and the downstream switchback roads (see fig. 13C). Specifically, we placed 24 seismometers at approximately 120-foot spacing along the crest road (fig 13B), and two seismometers at 120-foot spacing along the center of all three downstream switchback roads. Deployment layout with locations of clusters is shown in table 2.
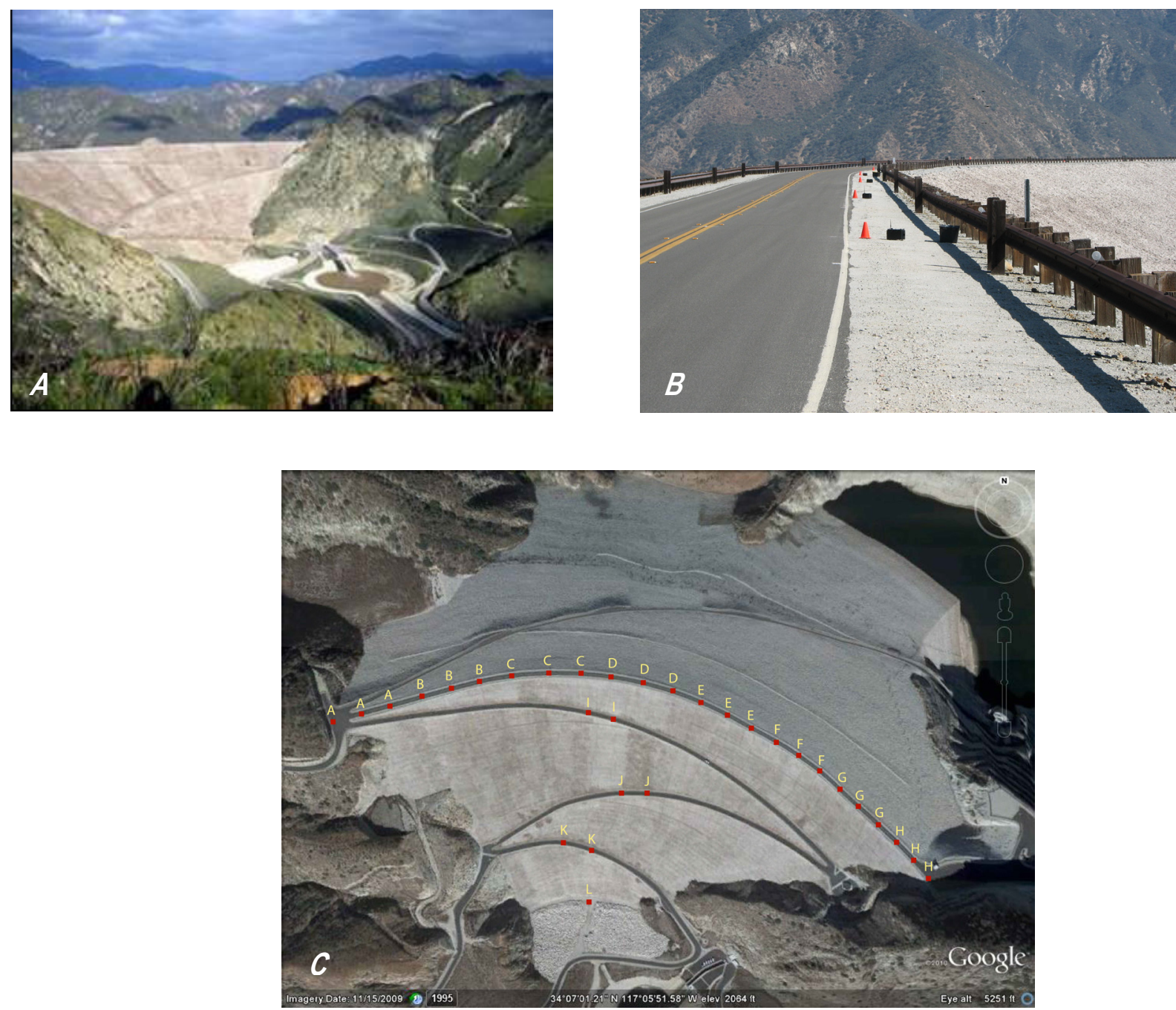

Figure 13. Seven Oaks Dam in Highland, California. A, Aerial view. Picture taken on February 8, 2005, by Steve Schumaker. $B$, Example ShakeBoxes deployed on crest of dam. $C$, Network configuration showing locations (red squares) of the 31 seismometers. 
Table 2. Locations of sensors and associated clusters during Seven Oaks Dam deployment. Sensors on crest road are listed in increasing distance order from westernmost to easternmost end and are approximately 120 feet apart. See also figure 13.

[\#, number]

\begin{tabular}{|c|c|c|}
\hline ShakeBox Serial \# & Cluster \# & Location \\
\hline B00E & A & Crest—westernmost end \\
\hline B008 & A & Crest \\
\hline B007 & $\mathrm{A}$ & Crest \\
\hline $\mathrm{B} 00 \mathrm{~A}$ & $\mathrm{~B}$ & Crest \\
\hline B00B & $\mathrm{B}$ & Crest \\
\hline B009 & $\mathrm{B}$ & Crest \\
\hline B00C & $\mathrm{C}$ & Crest \\
\hline $\mathrm{B} 00 \mathrm{~F}$ & $\mathrm{C}$ & Crest \\
\hline B00D & $\mathrm{C}$ & Crest \\
\hline $\mathrm{B} 010$ & $\mathrm{D}$ & Crest \\
\hline B012 & $\mathrm{D}$ & Crest \\
\hline B011 & $\mathrm{D}$ & Crest \\
\hline B020 & $\mathrm{E}$ & Crest \\
\hline B015 & $\mathrm{E}$ & Crest \\
\hline B014 & $\mathrm{E}$ & Crest \\
\hline B018 & $\mathrm{F}$ & Crest \\
\hline B016 & $\mathrm{F}$ & Crest \\
\hline B017 & $\mathrm{F}$ & Crest \\
\hline B019 & G & Crest \\
\hline B01B & G & Crest \\
\hline B01A & G & Crest \\
\hline B01E & $\mathrm{H}$ & Crest \\
\hline B01D & $\mathrm{H}$ & Crest \\
\hline B01C & $\mathrm{H}$ & Crest - easternmost end \\
\hline B02B & $\mathrm{I}$ & Middle of highest switchback road \\
\hline B02A & I & Middle of highest switchback road \\
\hline B029 & $\mathrm{J}$ & Middle of middle switchback road \\
\hline B028 & $\mathrm{J}$ & Middle of middle switchback road \\
\hline B022 & $\mathrm{K}$ & Middle of lowest switchback road \\
\hline B023 & $\mathrm{K}$ & Middle of lowest switchback road \\
\hline B02C & $\mathrm{L}$ & Base of dam \\
\hline
\end{tabular}


Spectra from several hours of ambient vibration data collected during one of the deployment days are shown in figure 14. This deployment posed problems we did not anticipate, alerting us to issues that still needed to be solved before making ShakeNet fully reliable. We had expected that wireless communication would be excellent because of the clear mote-to-mote line of site and because there were no wireless signals present that would interfere with ours. Communication between some motes and masters was faulty as it turned out, but we expect that more powerful antennas will partially solve this problem in the future. As with the previous deployment, the masters' hardware again posed problems for reliable data recording. During the data collection, we could not easily establish communication between the master and ShakeBoxes in at least four clusters. After trial-and-error placement of the antennas in various possible orientations, we were able to reach all ShakeBoxes, but postdeployment analysis showed that the communication links did not last long. We hypothesize that the reasons for bad communication links might be that the antennas we used were relatively weak for outdoor environments; they worked well in the 1100 Wilshire Boulevard building deployment which was indoors where walls helped reflect signals. We also encountered a strange reset behavior in the USB driver of the master nodes. Because of the reliability issue of the previous master (ebox) nodes used at the 1100 Wilshire Boulevard building, we replaced them with newer nodes with equivalent functionality and newer hardware (manufactured by Habey). The unpredictable resets occurred with three clusters, and we need to further test these to pinpoint the cause. It might be that these Habey nodes are faulty or that the master motes do not function well. The very small ambient vibration amplitudes of this large, stiff dam, near the internal noise level of the Colibrys sensors, precluded us from performing system modal identification from the 3-day records (fig. 14).

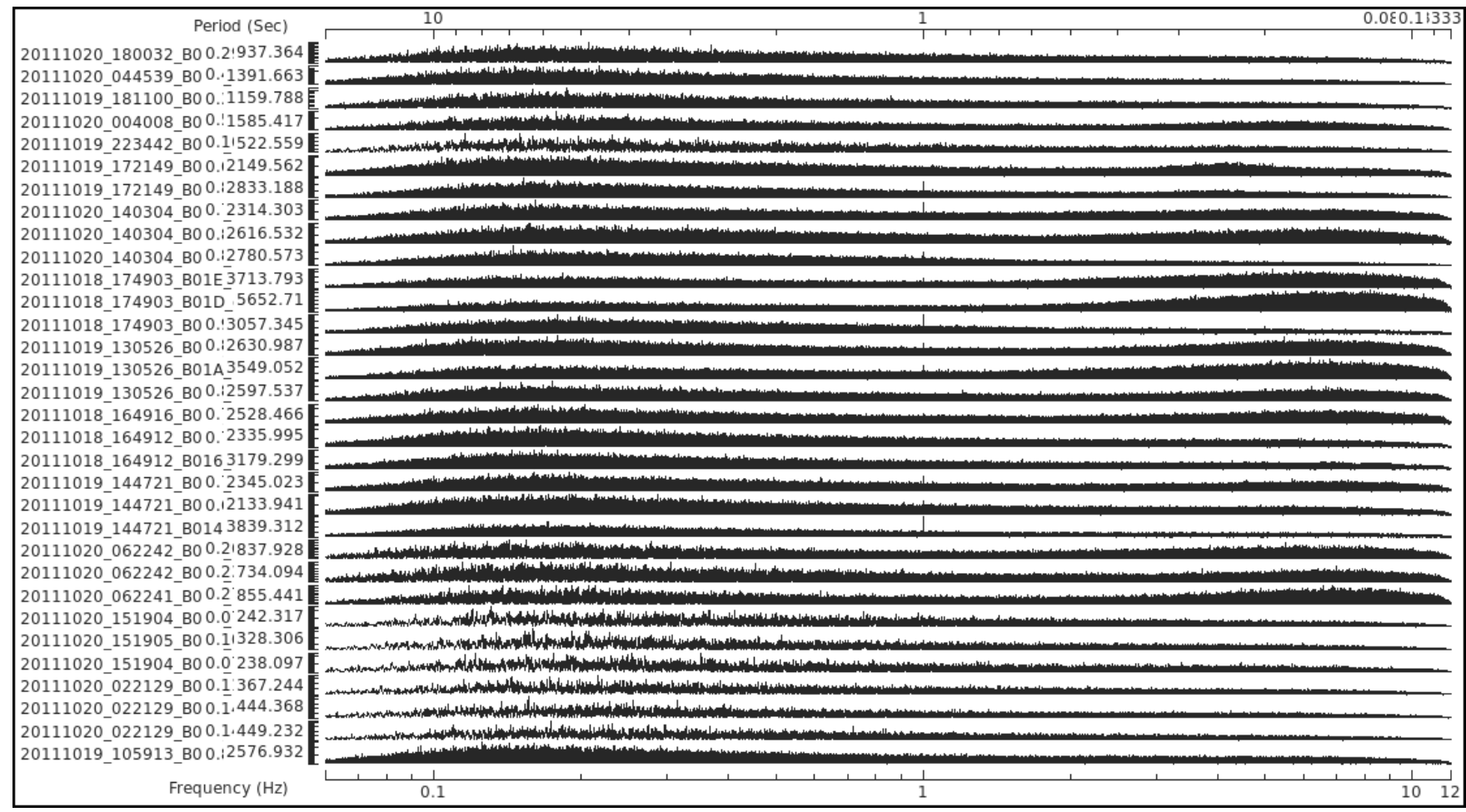

Figure 14. Ambient vibration spectra from transverse direction (channel 2, north-south orientation) produced from several hours of acceleration data recorded during one of the deployment days at Seven Oaks Dam in Highland, California. Numbers overwriting file name in left-hand column indicate amplitude normalization factor. 


\section{Summary and Conclusions}

The ShakeNet project enabled the testing of new wireless protocols with accelerometers to collect ambient vibration data in order to identify system response modes of civil structures. ShakeNet deployments required placing the nodes in harsh radio environments; thus, one of the most important aspects of the communication protocol development was to find the best route to the data collection node and to minimize packet drops. Another aspect of the development was that ShakeNet end-users not be required to write customized wireless sensor network data collection and communication software. Use of existing wireless sensor network software (Tenet) and modifying it for ShakeNet requirements helped reduce development time because Tenet could be tasked to operate a number of applications. The ShakeBoxes are currently being used by other researchers doing conducting software development so that the instruments can be operated either in network mode or in individual node mode in any Windows or Linux Operating System.

Since the conclusion of this project, new research has been carried out in seismic networks in which nodes are hosted by nontechnical volunteers. We envision that next-generation ShakeBoxes could be incorporated into such networks. The Quake-Catcher Network (QCN; Cochran and others, 2009, 2011) and Community Seismic Network (CSN; Clayton and others, 2011; Kohler and others, 2013) are dense networks of accelerometers deployed by community volunteers in homes, public spaces associated with civic services (such as city libraries), utilities, university campuses, and business offices. In addition to the standard USB device, which connects to the host's computer, a stand-alone version directly connects to the Internet via Ethernet. The QCN sensors in buildings are connected to netbooks with continuous data streaming in real time via the Berkeley Open Infrastructure for Network Computing (BOINC) software program to a server at Stanford University (Cochran and others, 2009). The basic analysis currently done uses only a fraction (1-5 percent) of the computing capabilities of the desktop, netbook, or other computer that is monitoring the sensor. Therefore, each sensor node has additional processing power that can be exploited for more than just the current event detection and data streaming. In the case of CSN, the data are sent to a cloud service where the data are fused (Clayton and others, 2011).

Future developments with community-hosted networks can take advantage of ShakeNet/ShakeBox setups by exploiting performance in the individual computers, or subsets of those computers, that are connected to the sensors, and cloud or distributed computing. Computations could be carried out on collections of sensors in a multi-tiered fashion (see Zhao and Guibas, 2004; Pottie and Kaiser, 2006; Iyengar and Brooks, 2012; Madisetti and Bahga, 2014) such that a subset of clients could aggregate information across only part of the structure, and one client (or stand-alone computer) could aggregate information for the entire building. This framework - combined with enhancements in telemetry systems that include, for example, cellular data-would provide increased management of the overall system in a wide variety of indoor and outdoor environments for structural monitoring.

\section{References Cited}

Advanced National Seismic System Structural Instrumentation Guideline Committee, 2005, Guideline for ANSS seismic monitoring of engineered civil systems: U.S. Geological Survey Open-File Report 2005-1039, 49 p.

Advanced National Seismic System Technical Integration Committee, 2007, Instrumentation guidelines for the advanced national seismic system: U.S. Geological Survey, 30 p., accessed March 30, 2015, at http://earthquake.usgs.gov/monitoring/anss/docs/ANSS_WGD_InstrGuideline_June2007.pdf. 
Celebi, M., Safak, E., and Youssef, N., 1991, Torsional response of unique building: Journal of Structural Engineering, v. 117, no. 5, p. 1549-1566.

Clayton, R., Heaton, T., Chandy, M., Krause, A., Kohler, M., Bunn, J., Olson, M., Faulkner, M., Cheng, M., Strand, L., Chandy, R., Obenshain, D., Liu, A., and Aivazis, M., 2011, Community Seismic Network: Annals of Geophysics, v. 54, no. 6, p. 738-747, doi:10.4401/ag-5269.

Cochran, E.S., Lawrence, J.F., Christensen, C., Jakka, R.S., 2009, The Quake-Catcher NetworkCitizen science expanding seismic horizons: Seismological Research Letters, v. 80, no. 1, p. 26-30, doi:10.1785/gssrl.80.1.26.

Cochran, E.S., Lawrence, J.F., Kaiser, A., Fry, B., Chung, A.I., Christensen, C., 2011, Comparison between low-cost and traditional MEMS accelerometers: a case study from the M7.1 Darfield, New Zealand, aftershock deployment: Annals of Geophysics, v. 54, no. 6, p. 728-737.

Gnawali, O., B. Greenstein, B., Jang, K-Y., Joki, A., Paek, J., Vieira, M., Estrin, D., Govindan, R., Kohler, E., 2006, The TENET architecture for tiered sensor networks, in Proceedings of the ACM Conference on Embedded Networked Sensor Systems, Boulder, Colo., October 31-November 3, 2006: New York, ACM, p. 153-166.

Iyengar, S.S., and Brooks, R.R., eds., 2012, Distributed Sensor Networks: (2d ed.): Boca Raton, Fla., CRC Press, $942 \mathrm{p}$.

Kohler, M.D., Davis, P.M., and Safak, E., 2005, Earthquake and ambient vibration monitoring of the steel frame UCLA Factor building: Earthquake Spectra, v. 21, no. 3, p. 715-736.

Kohler, M.D., Heaton, T.H., and Bradford, S.C., 2007, Propagating waves in the steel, moment-frame Factor building recorded during earthquakes: Bulletin of the Seismological Society of America, v. 97, no. 4, p. 1334-1345, doi:10.1785/0120060148.

Kohler, M.D., Heaton, T.H., Cheng, M-H., 2013, The Community Seismic Network and Quake-Catcher Network - enabling structural health monitoring through instrumentation by community participants, in Sensors and Smart Structures Technologies for Civil, Mechanical, and Aerospace Systems: Proceedings of the Society of Photo-Optical Instrumentation Engineers (SPIE) Conference, San Diego, Calif., March 10-14, 2013, v. 8692, 8 p.

Kohler, M.D., Heaton, T.H., Govindan, R., Davis, P., and Estrin, D., 2006, Using embedded wired and wireless seismic networks in the moment-resisting steel frame Factor building for damage identification: Proceedings of the $4^{\text {th }}$ China-Japan-U.S. Symposium on Structural Control and Monitoring, Hangzhou, China, October 16-17, 2006, 7 p.

Madisetti, V., and Bahga, A., 2014, The internet of things; a hands-on approach: Vijay Madisetti, 446 p. Nagayama, T., Spencer, Jr., B.F., and Rice, J.A., 2007, Structural health monitoring utilizing Intel's Imote2 wireless sensor platform, in Masayoshi, T., Yun, C-B., Giurgiutiu, V., eds., Sensors and Smart Structures Technologies for Civil, Mechanical, and Aerospace Systems 2007: Proceedings of the Society of Photo-Optical Instrumentation Engineers (SPIE) Conference, v. 6529, 15 p.

Pottie, G., and Kaiser, W., 2006, Principles of embedded network system design: Cambridge, U.K., Cambridge University Press, 548 p.

Werner-Allen, G.W., Lorincz, K., Johnson, J., Lees, J., and Welsh, M., 2006, Fidelity and yield in a volcano monitoring sensor network, in Proceedings of the USENIX $7^{\text {th }}$ Symposium on Operating Systems Design and Implementation, 2006: Berkeley, Calif., USENIX Association, p. 381-396.

Zhao, F., and Guibas, L., 2004, Wireless sensor networks-An information processing approach: San Francisco, Morgan Kaufmann Publishers, 376 p. 